\title{
Value network engagement and effects of memory-related processing during encoding and retrieval of value
}

\author{
Lindsay B. Conner ${ }^{\mathrm{a}}$ \\ Marilyn Horta ${ }^{b}$ \\ Natalie C. Ebner b, c, d \\ Nichole R. Lighthall a, ${ }^{*}$
}

\begin{abstract}
Author Note
${ }^{\text {a }}$ Department of Psychology, University of Central Florida, Orlando, FL, USA

${ }^{\mathrm{b}}$ Department of Psychology, University of Florida, Gainesville, FL, United States

${ }^{c}$ Department of Aging and Geriatric Research, Institute on Aging, University of Florida, Gainesville, FL, United States

${ }^{\mathrm{d}}$ Department of Clinical and Health Psychology, Center for Cognitive Aging and Memory, University of Florida, Gainesville, FL, United States

* Correspondence should be addressed to Nichole Lighthall, Department of Psychology, University of Central Florida, 4111 Pictor Lane, Orlando, FL 32816. Nichole.Lighthall@ucf.edu
\end{abstract}


VALUE NETWORK DURING ENCODING AND RETRIEVAL

\begin{abstract}
Decision makers rely on episodic memory to calculate choice values in everyday life, yet it is unclear how neural mechanisms of valuation differ when value-related information is encoded versus retrieved from episodic memory. The current fMRI study compared neural correlates of value while information was encoded versus retrieved from memory. Scanned tasks were followed by a behavioral episodic memory test for item-attribute associations. Our analyses sought to $(i)$ identify neural correlates of value that were distinct and common across encoding and retrieval, and (ii) determine whether neural mechanisms of valuation and episodic memory interact. The study yielded three primary findings. First, value-related activation in the frontostriatal reward circuit and posterior parietal cortex was comparable across valuation phases. Second, value-related activation in select fronto-parietal and salience regions was significantly greater at value retrieval than encoding. Third, there was no interaction between neural correlates of valuation and episodic memory. Taken with prior research, the present study indicates that fronto-parietal and salience regions play a key role in retrieval-dependent valuation and contextspecific effects likely determine whether neural correlates of value interact with episodic memory.
\end{abstract}

Keywords: value network, episodic memory, fMRI, decision making, encoding, retrieval 
VALUE NETWORK DURING ENCODING AND RETRIEVAL

\section{Value network engagement and effects of memory-related processing during encoding and retrieval of value}

Adaptive decision making involves the representation of choice values, and decision makers often rely on episodic memory to calculate choice values in everyday life. For example, consumers may rely on episodic memory to encode specific value-related information from product reviews, advertisements, or personal experiences, and retrieve this information from long-term storage when making purchasing decisions. As depicted in Figure 1, the neural substrates most commonly implicated in valuation include fronto-striatal "reward circuitry", particularly the ventromedial prefrontal cortex and ventral striatum (light blue regions; Bartra, McGuire, \& Kable, 2013; Brosch \& Sander, 2013; Clithero \& Rangel, 2014; Gläscher, Hampton, \& O’Doherty, 2009; Kable \& Glimcher, 2009; Levy \& Glimcher, 2012; Liu, Hairston, Schrier, \& Fan, 2011; Zhang, Larcher, Misic, \& Dagher, 2017). Additionally, more lateral regions of the orbitofrontal cortex support the integration of value information for goal-directed decision making (Nogueira et al., 2017; Wallis, 2007; Walton, Chau, \& Kennerley, 2015). Brain regions within the fronto-parietal "control network" have also been implicated in choice valuation. These include the posterior parietal, posterior cingulate, lateral prefrontal, and middle frontal cortices (dark blue regions; Bartra, McGuire, \& Kable, 2013; Clithero \& Rangel, 2014; Dosenbach, Fair, Cohen, Schlaggar, \& Petersen, 2008; Gläscher et al., 2012; Hare, Camerer, \& Rangel, 2009; Hsu \& Goh, 2016; Liu et al., 2011; Ptak, 2011; Sadaghiani \& D’Esposito, 2015). Finally, value processing has been associated with recruitment of the arousal-based "salience network" particularly the dorsal anterior cingulate cortex, anterior insula, thalamus, and lateral prefrontal cortex (red regions; Bartra, McGuire, \& Kable, 2013; Lamichhane \& Dhamala, 2015; Ploran et 
VALUE NETWORK DURING ENCODING AND RETRIEVAL

al., 2007; Sadaghiani \& D’Esposito, 2015; Seeley et al., 2007; Wallis, Stokes, Cousijn, Woolrich, \& Nobre, 2015).

\section{Figure 1}

Brain regions implicated in valuation

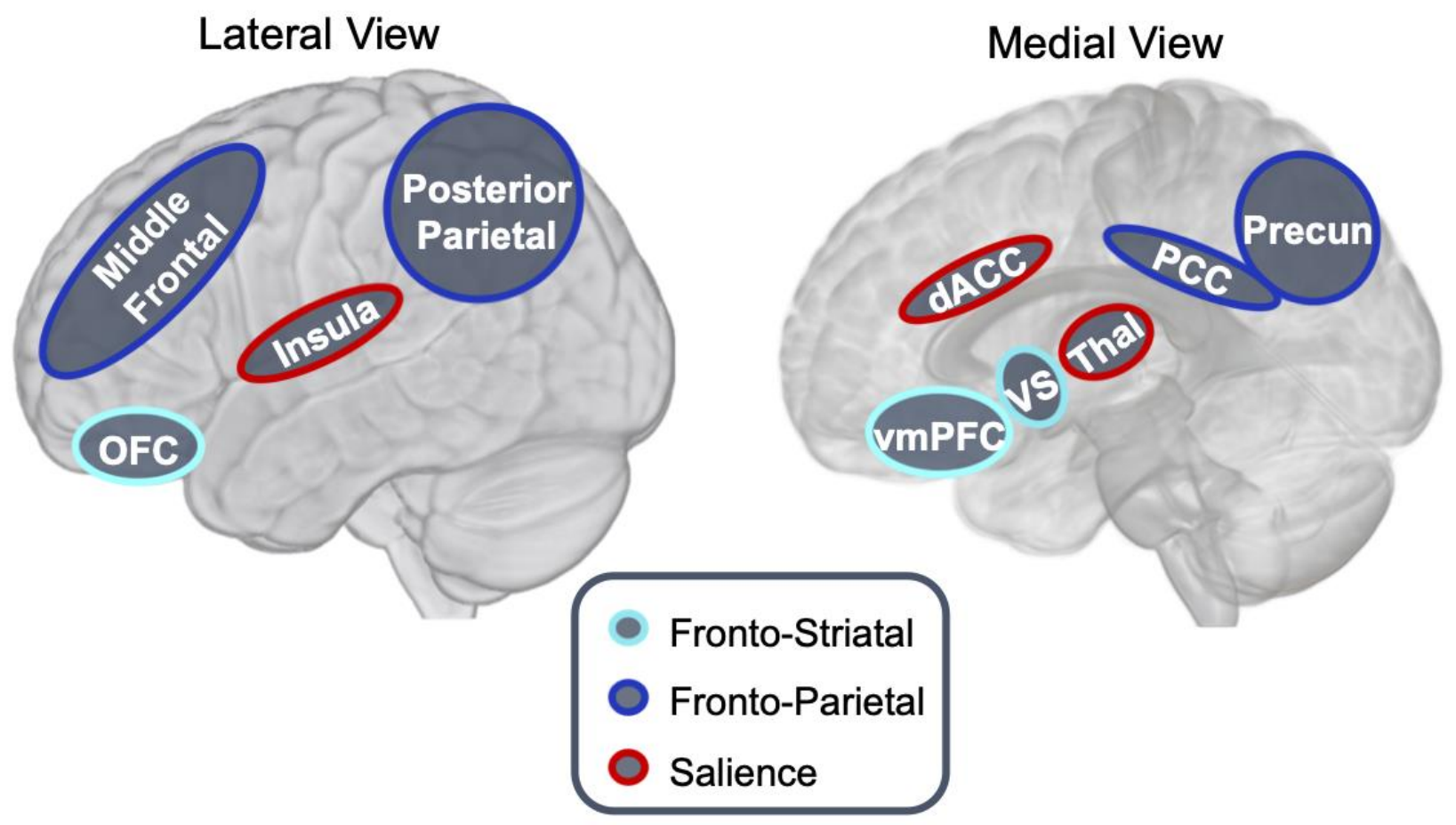

Note. Previous research has implicated a variety of brain areas spanning the fronto-striatal (in light blue), fronto-parietal (in dark blue), and salience networks (in red) in different aspects of value processing.

Abbreviations: OFC - orbitofrontal cortex; vmPFC - ventromedial prefrontal cortex; VS ventral striatum; dACC - dorsal anterior cingulate cortex; Thal - thalamus; PCC - posterior cingulate cortex; Precun - precuneus. 


\section{VALUE NETWORK DURING ENCODING AND RETRIEVAL}

Despite this wealth of research, and the fact that valued-based decision making frequently relies on episodic memory (Enkavi et al., 2017; Murty, FeldmanHall, Hunter, Phelps, \& Davachi, 2016; Shadlen \& Shohamy, 2016), it is presently unclear how encoding and retrieval processes affect the recruitment and interplay of regions in the value network. In particular, we are unaware of any studies that have directly examined common and distinct neural mechanisms of value encoding and value retrieval. Because of this gap, it is unclear if brain regions representing the "common currency" of value (Levy \& Glimcher, 2012) differ during encoding versus retrieval of value. Further, effective mapping of value mechanisms allow for better insight into the building blocks of optimal decision making. A central reason for this gap in the literature is that separate contributions of encoding and retrieval to value-based processing cannot be assessed by tasks commonly used to elicit neural value signals.

Specifically, studies of value processing often rely on reinforcement learning paradigms, in which stimulus- or action-outcome associations are learned implicitly and gradually from experience (Daw, Gershman, Seymour, Dayan, \& Dolan, 2011; Dickerson, Li, \& Delgado, 2011; Gershman, Pesaran, \& Daw, 2009; Gläscher, Daw, Dayan, \& O’Doherty, 2010; Gläscher, Hampton, \& O’Doherty, 2009; Knutson, Taylor, Kaufman, Peterson, \& Glover, 2005; Montague, King-Casas, \& Cohen, 2006; Palminteri, Boraud, Lafargue, Dubois, \& Pessiglione, 2009; Rushworth \& Behrens, 2008; Rushworth, Noonan, Boorman, Walton, \& Behrens, 2011; Valentin, Dickinson, \& O’Doherty, 2007; Wimmer, Braun, Daw, \& Shohamy, 2014; Wimmer, Li, Gorgolewski, \& Poldrack, 2018). One-shot decision tasks are also commonly employed to assess neural correlates of value. In these tasks, decisions are made from information presented simultaneously during, or immediately preceding, choice (e.g., potential rewards, outcome probabilities, reward delays; Carter, Meyer, \& Huettel, 2010; Clithero, Carter, \& Huettel, 2009; 
VALUE NETWORK DURING ENCODING AND RETRIEVAL

Jimura, Chushak, \& Braver, 2013; Kable \& Glimcher, 2007; Koscik, Man, Jahn, Lee, \&

Cunningham, 2020; Levy \& Glimcher, 2012; Levy, Snell, Nelson, Rustichini, \& Glimcher, 2010;

McClure, Laibson, Loewenstein, \& Cohen, 2004; Padoa-Schioppa \& Assad, 2006; Peters \&

Büchel, 2009; Shenhav, Rand, \& Greene, 2017; Tom, Fox, Trepel, \& Poldrack, 2007).

While these paradigms do not explicitly examine the influence of episodic memory

processes, it can be argued that both types of valuation tasks include features of encoding comprising the acquisition and integration of available information for subsequent cognitive processes. Thus, dissociating value encoding and retrieval is not possible in these paradigms. Further, what is currently considered the canonical valuation network may be more reflective of value encoding than value retrieval.

There is also a lack of clarity about whether and how valuation and episodic memory processing may interact. Some studies have suggested competition between value and episodic memory systems, while others suggest cooperation or more independent, non-interactive processing. Resolving these mixed findings is critical for determining situations in which engagement of memory processing will impair or facilitate value-based decision making. With respect to findings of memory and valuation competition, when value learning takes place over many prior experiences, select and incidental episodic memory for past choices can result in unequal weighting of prior experiences and biased decision making (Bhui, 2018; Bornstein, Khaw, Shohamy, \& Daw, 2017; Bornstein \& Norman, 2017; Wimmer et al., 2014). For example, a gamble option may yield a net gain over many selections - but if one past loss is more available to episodic memory, the value of the choice option is likely to be underestimated. This kind of decision bias is predicted by the degree to which previously experienced learning contexts trigger neural reinstatement (Bornstein \& Norman, 2017). 
VALUE NETWORK DURING ENCODING AND RETRIEVAL

Neural mechanisms of these effects further suggest competition between valuation and memory processes during reward-guided reinforcement learning. Indeed, neural responses to value-learning contexts in the parahippocampal place area predict greater influence of past experiences from that context (Bornstein \& Norman, 2017), and greater episodic memory for stimuli presented at choice predict weaker predictor error signals in the functionally connected striatum (Wimmer et al., 2014).

In contrast, evidence of cooperation includes behavioral findings that encoding and retrieval of associated episodic information facilitate value-based decision making in novel situations and when choice valuation is based on limited prior experience. For example, when choice values are encoded via discrete experiences (episodic encoding of value), memory for salient choiceassociated stimuli predicts value-based decision performance (Duncan \& Shohamy, 2016; Murty et al., 2016). Such findings have led to the proposal that familiar stimuli can trigger a "retrieval state" in which value information from associated episodic memories are more easily recollected (Duncan \& Shohamy, 2016).

While these studies did not examine neural mechanisms, a conceptually related line of neuroimaging studies have examined the neural correlates of value inferences from stored representations of relevant associations. Such studies find related recruitment of the orbitofrontal or ventromedial prefrontal cortices alongside functionally connected hippocampal regions (Jones et al., 2012; Petrides, 2007; Richter, Chanales, \& Kuhl, 2015; Spalding et al., 2018; Stalnaker, Cooch, \& Schoenbaum, 2015; Zeithamova, Dominick, \& Preston, 2012). Also relevant are studies of episodic encoding tasks with variation in stimuli values. For example, one study demonstrated that increased engagement of semantic processing regions was associated with selective encoding of high-value words during an episodic memory task, suggesting stimulus 
VALUE NETWORK DURING ENCODING AND RETRIEVAL

value effects on level of processing (Cohen, Rissman, Suthana, Castel, \& Knowlton, 2014).

Other studies suggest that medial temporal regions are more responsive to novel high-value stimuli, with distinguishable engagement of anterior and posterior hippocampal regions, respectively, for processing novel information versus recognizing and retrieving repeated information (Kuhl, Shah, DuBrow, \& Wagner, 2010; Poppenk, McIntosh, Craik, \& Moscovitch, 2010; Ritchey, Wing, LaBar, \& Cabeza, 2013; Wolosin, Zeithamova, \& Preston, 2013).

Thus, a number of regions supporting associative memory appear to be sensitive to stimuli values. It is still unclear, however, whether the neural mechanisms of episodically encoded value have a cooperative relationship with episodic memory mechanisms (e.g., enhanced value signal with greater memory for associates) or whether the two systems function independently (e.g., non-interacting neural correlates). Our study attempts to address this remaining knowledge gap on the relationship between value and memory mechanisms.

The present study contributes to our understanding of valuation-memory intersections by comparing the neural mechanisms of value-based judgments when relevant information is present (during encoding) versus when it is absent and must be retrieved from episodic memory (during retrieval). During the study task, participants made value-related judgments about different products based on "consumer reviews" presented an encoding phase and a retrieval phase, while undergoing functional magnetic resonance imaging (fMRI). This design allowed for identifying the neural mechanisms of valuation unique to, and common across, value encoding and value retrieval (Fig. 2a). Additionally, our study task manipulated encoding experiences by presenting products with either one or two consumer reviews at encoding and assessed memory for product attributes using a behavioral memory recognition test. These task features allowed for examining the relationship between valuation and episodic memory systems (Fig. 2b). 
VALUE NETWORK DURING ENCODING AND RETRIEVAL

Based on findings using probabilistic learning and one-shot decision paradigms, we expected that recruitment of regions most commonly implicated in valuation (e.g., fronto-striatal regions) would be more characteristic of valuation at encoding while participants actively acquired and integrated information. As our task involved discrete encoding of value in an episodic context, we expected to find evidence of cooperation or independence, rather than competition, between valuation and episodic memory mechanisms. 
VALUE NETWORK DURING ENCODING AND RETRIEVAL

Figure 2

Visualization of study goals

A Goal 1: Distinct \& Common Valuation Mechanisms

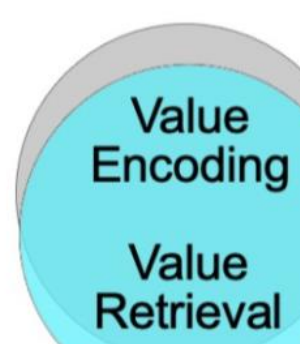

Common

Mechanisms
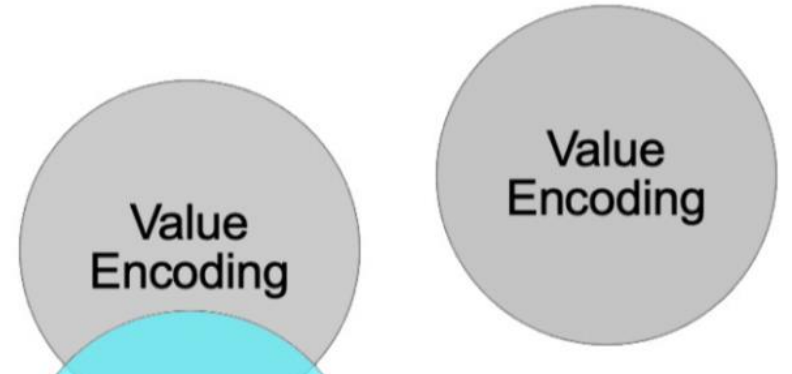

Value

Retrieval

B

Goal 2: Independent vs. Interacting Valuation \& Episodic Memory Systems

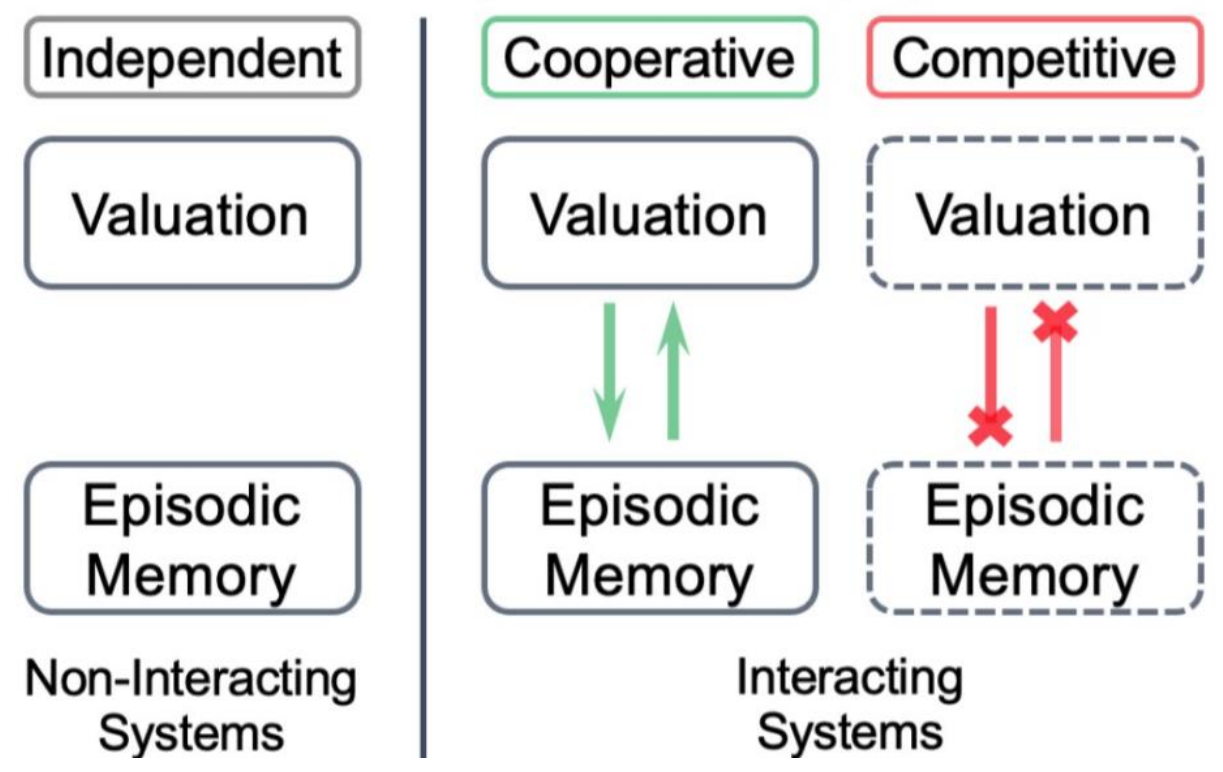


Note. (A) The primary study goal was to identify distinct and common neural mechanisms of valuation across value encoding and retrieval. To address this goal, brain activation associated with value for consumer products was determined while value-related information was present (value-encoding phase), and later, when value-related information was absent (value-retrieval phase). Resulting phase-subtraction and conjunction-based ROI masks were used to determine whether value-related activity was significantly distinctive during value encoding and retrieval. (B) The secondary study goal was to test for evidence of interaction versus independence between neural mechanisms of valuation and episodic memory. In the case of interaction, we then aimed to determine the nature of the relationship between these systems by probing for competition versus cooperation. To address this goal, demands on memory was manipulated at encoding and retrieval, with corresponding neuroimaging analyses examining effects of encoding experiences on value-related brain activation. Additional analyses were used to determine whether brain activation related to value differed with successful encoding of associated episodic details (product attributes). An absence of memory-dependent effects on the neural correlates of value was taken as evidence for independence. Enhanced value-related activation with more encoding experiences or successful episodic encoding was taken as evidence of cooperation (reciprocal green arrows). Diminished value-related activation with more encoding experiences or successful episodic encoding was taken as evidence of competition (inhibiting red lines). 
VALUE NETWORK DURING ENCODING AND RETRIEVAL

\section{Materials and Methods}

\subsection{Participants}

Twenty-seven young adults were recruited from the University of Florida (UF) student population for participation in this fMRI study. Given the novel task and associated lack of prior results for power calculation, the sample size was based on guidelines by Simmons et al. (2011) and Thirion et al. (2007). All participants were healthy, right-handed, fluent in English, free of MRI contraindications, and had normal or corrected-to-normal vision (using MR-compatible eyeglasses). One participant was excluded from data analysis due to excessive head motion across multiple scans, along with four participants for technical errors during scanning or data transfer. Two additional participants were excluded due to failing task performance criteria (i.e., memory false alarm rate $\geq 3 S D$ s above the mean; responding with single value rating throughout the session). Our final sample included twenty participants $\left(M_{\text {Age }}=22.55, S D=3.72 ; 18-31\right.$ years old; 9 females).

\subsection{Procedure}

Participants were recruited from a psychology student database or via flyer, phone call, or email contact from participant registries. Prior to study enrollment, a phone screening was conducted to determine participant interest and eligibility. Data collection took place at the McKnight Brain Institute on the UF Gainesville campus. On the scan day, participants provided written informed consent and completed questionnaires that assessed demographic, health, and psychosocial factors (e.g., affect, motivation, and decision-making style; data not reported here). Participant compensation consisted of either course credit or a \$20 gift card depending on participant preference, with an additional $\$ 10$ bonus paid to all participants (see section 2.3.4. 
VALUE NETWORK DURING ENCODING AND RETRIEVAL

Incentive structure). UF and University of Central Florida (UCF) Institutional Review Boards approved the study.

\subsection{Consumer Judgment Task}

At session start, participants responded to questionnaires, reviewed task instructions, and completed a set of practice trials on a computer. After entering the scanner, participants reviewed task instructions again and practiced use of a four-button response box. After anatomical and resting-state scans, participants completed three blocks of the value-encoding task (10.02 min, 80 trials each) and two blocks of the value-retrieval task (9.26 min, 75 trials each) during fMRI. Immediately after the value-retrieval task, participants exited the scanner and completed a selfpaced behavioral memory test on product attributes from the encoding phase $(M=15.95 \mathrm{~min}, S D$ $=2.37 \mathrm{~min}, 390$ trials). Participants then responded to a questionnaire about their experience in the study and task-performance strategies. At the end of the session, participants received their compensation and were debriefed.

\subsubsection{Stimuli}

The stimuli used in this task were created to resemble common consumer products from a popular online shopping website. Each product was represented by an image and a product name (e.g., "Clock"). During the encoding phase, products were presented with associated "consumer reviews." Text was derived from real consumer reviews of the product rated as "most helpful" from the same online shopping website. For each product, review excerpts were taken from three comments that focused on different product attributes (e.g., battery life, ticking sound, number size). These attribute-specific comments were edited to fit three valence conditions: positive (e.g., "Quiet, hardly noticeable tick.”), negative (e.g., "Extremely loud ticking noise.”), and neutral (e.g., "Audible tick."). To confirm that each consumer review reflected its valence 
VALUE NETWORK DURING ENCODING AND RETRIEVAL

category, valence ratings were collected from a separate sample via Amazon Mechanical Turk

(MTurk). Individual MTurk raters assessed attribute-specific consumer reviews for only one valence condition. Review stimuli received 10 ratings each. Stimuli in the fMRI study were presented against a white background using E-Prime 2.0 software (Psychology Software Tools, Pittsburgh, PA) on a screen in the scanner room.

\subsubsection{Value encoding}

To index value at encoding and address our primary study goal, participants were asked to rate the valence of each consumer review while undergoing fMRI (Fig. 3a). Ratings were given on a discrete valence scale from 1 (negative) to 4 (positive) while the product was visible on the screen for 4 seconds. A black fixation cross was shown when the participant responded in time. Participants were informed that, if unable to respond within the allotted time, they could respond for an additional 2 seconds while a red ' $\mathrm{X}$ ' was on the screen. Failure to make any button press resulted in the trial being coded as a non-response; non-response trials were not included in the data analysis $(\sim 6 \%)$. To address trials with multiple button presses, the last response was used for the review rating. A jittered inter-stimulus interval (ISI) of 2 to 4 seconds (e.g., pseudorandom at discrete intervals of $250 \mathrm{~ms}$ ) occurred between each product.

Toward our secondary study goal, we manipulated the need for integration of value-related information during retrieval to probe demands on the memory system. Correspondingly, products varied in the number of encoding experiences such that products were presented with either one consumer review or two different reviews. In the one-experience condition, products were presented with either a positive or negative review. Products in the two-experience condition were presented twice within 2 to 4 encoding trials and were accompanied by two different attribute-specific reviews. In the two-experience condition, products were presented 
VALUE NETWORK DURING ENCODING AND RETRIEVAL

with either two reviews of opposing valence (negative followed by positive, positive followed by negative) or two neutral reviews. Ten products were presented per valence sequence category in each value-encoding block.

\section{Figure 3}

Consumer Judgment Task design

A

Task 1: Value Encoding (fMRI) Value Encoding Rating 1

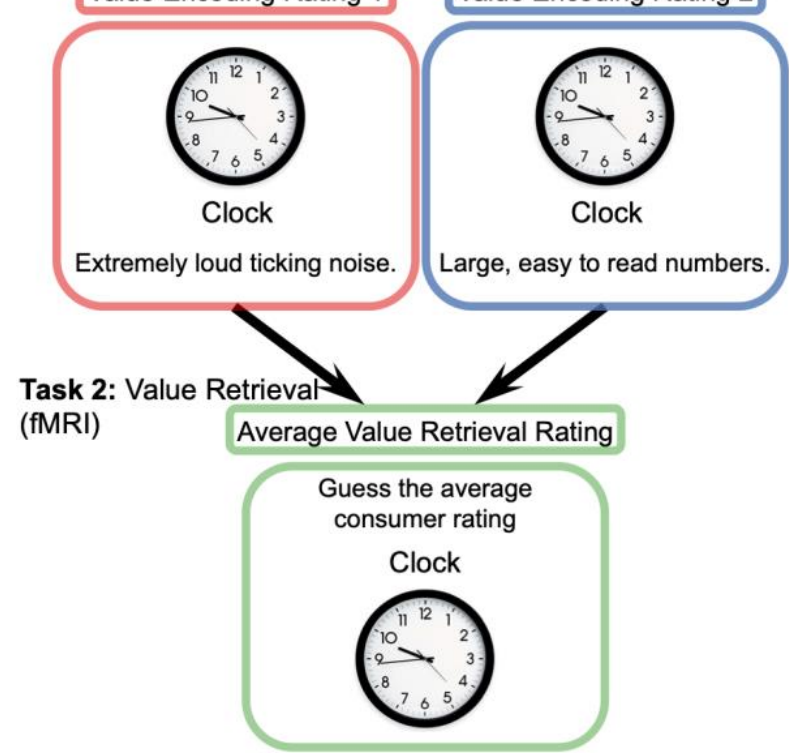

$\mathrm{B}$ Task 3: Attribute Memory (behavioral)

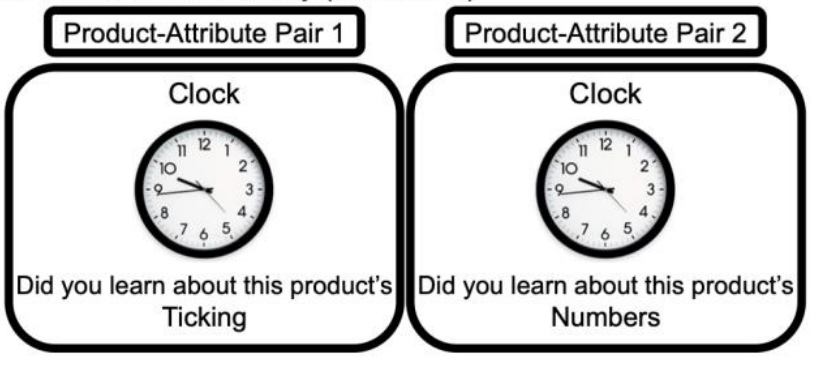

Foil Product-Attribute Pair

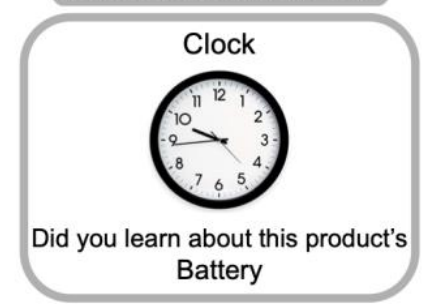

Note. The Consumer Judgment Task was divided into three phases: value encoding, value retrieval, and attribute memory. Value encoding and retrieval phases were completed during fMRI; the attribute memory task followed outside the scanner. (A) During value encoding, reviews of consumer products were rated using a discrete 1 (negative) to 4 (positive) value scale. Products were either presented with one consumer review or two different consumer reviews. During value retrieval, the average rating for each product was estimated based on the previously 
VALUE NETWORK DURING ENCODING AND RETRIEVAL

encountered consumer reviews, using a continuous 1 (negative) to 5 (positive) rating scale. (B)

Attribute memory was tested using a 1 (definitely yes) to 4 (definitely not) scale as to whether attribute-product pairs were presented together during encoding.

\subsubsection{Value retrieval}

To index value at retrieval and further address our primary study goal, participants were asked to estimate the average consumer rating of each product from the encoding phase on a continuous 1 (negative) to 5 (positive) scale based on the previously encountered consumer reviews while undergoing fMRI (Fig. 3a). Product ratings from the one-experience condition required the retrieval of only a single consumer review from recent memory. Ratings for products in the two-experience condition required retrieval of information from two different consumer reviews, adding the need to integrate previously encountered value information.

A continuous rating scale was used with a value range that differed from the discrete scale used in the encoding phase, such that rating consistency across phases would require a numerical translation for products in both the one- and two-experience conditions. Consistency in value ratings therefore required more than a simple repeated response across the encoding and retrieval phases. Products from value encoding were presented in random order during value retrieval. Retrieval trials began with the rating marker at the neutral position ('3’), and participants were given 4 seconds to respond on the slider scale, using their index and middle fingers to indicate increasingly negative or positive ratings, respectively. To make an exact rating of ' 3 ', participants pressed a key with their ring finger. Failure to press any button resulted in the trial being coded as a non-response; non-response trials were not included in the data analysis $(\sim 3 \%)$. 
VALUE NETWORK DURING ENCODING AND RETRIEVAL

\subsubsection{Incentive structure}

Participants were told that the amount of their payout bonus would be calculated based on the value of two products randomly selected at the end of the experiment. The task's incentive structure was tied to valuation in two ways. First, participants were told that trials (products) with more positive consumer reviews had the potential to yield more monetary gain, such that the most valuable products were associated with $\$ 5$ rewards and least valuable products with $\$ 1$ rewards. They were told that an external sample had rated all products based on the presented consumer reviews on a 1-5 scale and the average of those ratings determined the value of each product (i.e., \$1-5). This incentive structure parallels naturalistic memory-based consumer decision making, such that consumers are incentivized to remember high-value products due to their greater reward potential (utility), but memory for low-value products is also important as it helps consumers avoid poor choices.

Second, participants were told that their final bonus depended on correspondence between their ratings and the external sample's average ratings. As the participants were told the external sample's ratings were based on the same consumer reviewer comments they saw in the task, participants were incentivized to give ratings that reflected the product reviews. Specifically, participants were told that any discrepancy between their rating and the external sample's rating for a given product would be subtracted from the product's value. For example, if the external sample gave a product an average rating of ' 3 ' (worth \$3) and the participant guessed that the average rating was a ' 4 ' (valuation error of $1=\$ 1$ ), that product would only add $\$ 2$ toward their earnings if selected for the bonus. Notably, this incentive structure penalizes over- and underestimations of value, ensuring that participants had a vested interest in attending to, and correctly retrieving, information from all consumer reviews when making their ratings. As participants 
VALUE NETWORK DURING ENCODING AND RETRIEVAL

were told that all products were equally likely to be selected for the bonus, performance on each

trial was incentivized. At the end of the study, trials were not randomly selected and all participants received the maximum possible bonus of $\$ 10$.

\subsubsection{Episodic memory for product attributes}

Toward addressing our secondary goal, participants completed a memory test for product attributes outside the scanner, immediately after the value-retrieval phase. Each self-paced memory trial included the picture and name of a product presented during value encoding, along with a non-affective attribute (e.g., battery life, ticking sound, number size; Fig. 3b). Participants provided responses reflecting the degree to which they remembered specific attributes mentioned in the consumer reviews for individual products using a discrete 1 (definitely yes) to 4 (definitely not) scale. Each pairing of product and attribute encountered during the encoding phase was presented during the attribute memory phase, intermixed with foil attributes (one per product) to distinguish between false alarms and correct recognitions.

\subsection{Behavioral data analysis}

Behavioral data were analyzed using SPSS Statistics 25 (IBM Corp., RRID:SCR_002865). Valence ratings from encoding and retrieval were used to index valuation. To facilitate comparisons across encoding and retrieval ratings, transformations were applied to both measures to put them on $0-1$ scales. The transformation for encoding responses converted the original 1-4 interval scale into four equally spaced values ranging from $0-1$. Retrieval responses were converted from the original 1-5 continuous scale into z-score values. Because participants did not use the full range of the original retrieval scale, z-scored retrieval responses were based on the highest and lowest responses entered by each participant within a block. Z-scored retrieval ratings were then transformed to a 0-1 scale. Transformed ratings were used to calculate value- 
VALUE NETWORK DURING ENCODING AND RETRIEVAL

retrieval accuracy, which corresponded to the similarity between product-specific ratings at encoding and retrieval (i.e., encoding rating - retrieval rating). For products with two encoding experiences, value-retrieval accuracy calculations used the average of the two product-specific encoding ratings. Finally, episodic memory for product attributes was indexed using corrected recognition scores (\% hit rate - \% false alarm rate). Ratings of 1 (definitely yes) or 2 (maybe yes) for correct product pairings encountered in the attribute memory task phase were coded as hits, while the same ratings for incorrect ("foil") product pairs were coded as false alarms.

\subsubsection{Value rating behavior}

To establish task validity, we used a repeated measures analysis of variance (RM-ANOVA) to assess consistency between participant's value ratings at encoding and the three preset valence conditions (negative, neutral, and positive) for consumer reviews. Next, we utilized a linear mixed-effects model to verify the correspondence in value ratings at encoding and later retrieval and assess the effects of the number of encoding experiences on value retrieval. The linear mixed-effects model included subject as a random effect, with a random intercept for each subject, a restricted maximum likelihood estimation, and an unstructured covariance type. We conducted additional analyses to confirm that the unique consumer reviews presented with twoexperience products during encoding both contributed to later retrieval ratings and determined the relative influence of first and second encoding trials on subsequent value-retrieval ratings (see Supplementary Material, Section I).

\subsubsection{Value retrieval and episodic memory performance}

Toward our secondary goal of examining interactions between valuation and memory systems, we assessed episodic memory for product attributes. We first conducted a one-sample ttest on participants' corrected recognition scores to confirm that performance was above chance. 
VALUE NETWORK DURING ENCODING AND RETRIEVAL

We then utilized a trial-level linear mixed-effects model to gauge the potential interaction between value-retrieval accuracy and memory accuracy for each product, using hit rate as a representation of attribute memory performance and modeling subject as a random effect.

Additional analyses were conducted to confirm that the number of encoding experiences did not alter the correspondence between value ratings and attribute memory strength (see Supplementary Material, Section II).

\subsection{Neuroimaging data acquisition}

Brain images were acquired with a Philips Achieva 3T scanner using a 32-channel RF head coil. Participants viewed the experiment screen via a mirror placed inside of the head coil and used their right hand to respond via an MR-compatible four-button response box. Anatomical image acquisition involved a T1-weighted 3D localizer series (MPRAGE sequence), with 176 axial oblique slices (TR: $7.1 \mathrm{~ms}$; TE: $3.2 \mathrm{~ms}$; FOV: $240 \mathrm{~mm}^{2}$; flip angle: $8^{\text {o; }}$, voxel size: $1 \mathrm{~mm}^{3}$; scan time: $4.5 \mathrm{~min}$ ). After the anatomical scan, functional images were acquired using a singleshot Fast Field Echo-Planar Imaging (FFE-EPI) sequence (sensitivity-encoded [SENSE] for 2fold acquisition speed) sensitive to the blood oxygenation level-dependent (BOLD) signal (TR: 2000 ms; TE: 30 ms; FOV: 252 mm²; flip angle: 90º 38 interleaved transverse slices; voxel size: $3.5 \mathrm{~mm}^{3}$; no slice gap; acquisition matrix: 72 x 72 voxels; SPIR fat suppression). Functional scanning included a resting-state functional scan (not reported here), followed by three runs of the encoding task (295 TRs each) and two runs of the retrieval task (277 TRs each). Four acquired volumes from the start of each functional run were discarded prior to data analysis.

\subsection{Neuroimaging data analysis}

Structural and functional image analyses were conducted using FMRI Expert Analysis Tool (FEAT), from FMRIB's Software Library (FSL v6.0.1; RRID:SCR_002823; Smith et al., 2004). 
VALUE NETWORK DURING ENCODING AND RETRIEVAL

Image preprocessing steps included: $(i)$ motion correction with MCFLIRT (FMRIB's Motion

Correction Linear Image Registration Tool); (ii) spatial smoothing with a 5-mm full-width halfmaximum (FWHM) Gaussian kernel registered to the first image; (iii) high-pass temporal filtering equivalent to $100 \mathrm{sec}$; and (iv) skull stripping of structural images with BET (FMRIB's Brain Extract Tool). Registration was performed with FLIRT (FMRIB's Linear Image Registration Tool) such that each functional image was registered to both the participant's highresolution brain-extracted structural image (6 df) and the FSL Montreal Neurological Institute (MNI) template using an affine transformation (12 df). All scans included in the analysis had less than $3 \mathrm{~mm}$ relative head motion and were free of severe artifacts (e.g., spiking, ghosting, radio frequency noise, signal inhomogeneity).

All reported Z-statistic maps survived cluster-wise whole-brain correction with a clusterforming threshold of $\mathrm{Z}>2.3$ and cluster-correction at $p=.05$ (Worsley, 2001). To reduce the proportion of false positives identified within our group-level activation clusters and develop spatially constrained regions-of-interest (ROIs) for comparison of regions with robust valueassociated activity at encoding and retrieval, we further applied voxel-wise false discovery rate (FDR) multiple comparison corrections at a threshold of $q=.05$ to group-level contrast images (FSL-FDR; Genovese, Lazar, \& Nichols, 2002; Woo, Krishnan, \& Wager, 2014). Image coordinates are presented in MNI space and anatomical regions were identified using the Harvard-Oxford cortical and subcortical structural atlases (Makris et al., 2006). For visualization, Z-statistic maps are presented over an MNI-152 standard-space T1-weighted average structural template image with $2 \mathrm{~mm}$ resolution using the FSL image viewer (FSLeyes v0.27.3; McCarthy, 2020). ROI masks are rendered over a 3D MNI-152 standard brain using MRIcroGL (v1.2; Rorden, Karnath, \& Bonilha, 2007) with partial transparency set to relay depth information. 
VALUE NETWORK DURING ENCODING AND RETRIEVAL

Three value-encoding and two value-retrieval phase scans (Fig. 3a) were analyzed per participant using general linear models (GLM) with local autocorrelation correction (FMRIB's Improved Linear Model prewhitening). Trial events were convolved with a double-gamma hemodynamic response function and motion parameters were included as nuisance regressors. Trials with missing rating responses were not modeled ( $\sim \%$ of encoding and $3 \%$ of retrieval trials). Subject-level analyses used a fixed-effects model to combine data across runs for each participant. Group-level analyses used a mixed-effects model to combine data across participants, allowing for the examination of group means.

\subsubsection{Distinct and common neural correlates of value encoding and retrieval}

2.6.1.1. Value encoding network. We applied a GLM to fMRI data from the encoding phase (Fig. 3a) to identify BOLD responses associated with value at encoding. The encoding analysis included two trial events modeled by time of onset and subject response time $(\leq 4 \mathrm{sec})$ for event duration: $(i)$ encoding trials and (ii) encoding trials with associated value ratings as a parametric modulator orthogonalized to the main encoding trial regressor. The main contrast of interest tested the parametric effect of encoding value rating, representing the value network during encoding.

2.6.1.2. Value retrieval network. To identify BOLD response associated with value at retrieval, we applied a GLM to fMRI data from the retrieval phase (Fig. 3a) with events modeled by time of trial onset and the full trial duration $(\sim 4 \mathrm{sec})$. The retrieval analysis included four trial events: $(i)$ retrieval trials for one-experience products, (ii) retrieval trials for one-experience products with associated value ratings as a parametric modulator orthogonalized to the main retrieval trial regressor, (iii) retrieval trials for two-experience products, and (iv) retrieval trials for two-experience products with associated value ratings as a parametric modulator 
VALUE NETWORK DURING ENCODING AND RETRIEVAL

orthogonalized to the main retrieval trial regressor. The main contrasts of interest tested $(i)$ the parametric effect of retrieval value rating, (ii) the bidirectional effects of one versus two encoding experiences, and (iii) bidirectional effects of encoding experiences weighted by retrieval value ratings. Together with the above analyses of fMRI data at encoding, these analyses addressed our two central study goals. Full whole-brain value-related activation results are available in the Supplementary Materials under Section III.

\subsubsection{Distinct and common neural correlates of value encoding and retrieval. Toward} our primary goal, we created binarized masks of group-level FDR-corrected parametric activity related to value ratings at encoding and retrieval. To identify distinct neural correlates of value during encoding and retrieval, we conducted bidirectional contrasts of valuation masks between valuation phases. The resulting phase-subtraction masks (i.e., Encoding > Retrieval; Retrieval > Encoding) represented regions exhibiting significant value-related activation in only one phase encoding or retrieval. To identify common neural correlates of value across phases, a conjunction analysis was conducted using the aforementioned masks of value-related activity from each phase. The resulting conjunction map represents areas where value-related activations were significant during both encoding and retrieval (i.e., Encoding x Retrieval; Biswal et al., 2010; Friston, Holmes, Price, Büchel, \& Worsley, 1999).

To test whether activity differed significantly by value-encoding versus value-retrieval phase within the resulting phase-subtraction and conjunction clusters, we compared mean activity levels between valuation phases in the resulting ROI clusters. Mean percent BOLD signal change values were extracted from subject-level value-weighted contrast images for encoding and retrieval within binarized masks from the phase-subtraction and conjunction clusters using FSL featquery. Included ROIs represented regions previously implicated in valuation (Fig. 1), with 
VALUE NETWORK DURING ENCODING AND RETRIEVAL

regions associated primarily with sensorimotor functions excluded from the ROI analysis. To compare ROI activity levels between value-encoding and value-retrieval phases, separate twoway RM-ANOVAs (valuation ROIs x valuation phases) with Greenhouse-Geisser sphericity correction were conducted for each group of phase-subtraction and conjunction ROIs. Post-hoc pairwise comparisons were conducted with Bonferroni-Holm correction for multiple comparisons to assess significant ROI-phase relationships.

Additional analyses were conducted to examine effects of the encoding experience manipulation. First, a control analysis was conducted to address the possibility that repeated presentation of products in the two-experience condition caused memory interference effects that impacted our observed results for value-related activity. This analysis examined value-related activity at encoding for products in the one-experience condition alone in order to compare them with our main analysis of value correlates across encoding experience conditions. Then, to address our secondary study goal, analyses were conducted to examine the impact of multiple encoding experiences on value-related brain activation (e.g., memory demand from information integration). In brief, the encoding experience manipulation did not yield effects on value-related activation that survived FDR correction, consistent with an independence of valuation and memory systems. More detailed methods and results of these analyses are available in the Supplementary Materials under Sections IV and V.

\subsubsection{Relationship between valuation and episodic memory systems}

Toward our secondary study goal, we next conducted a GLM analysis to determine if valuerelated activation depended on whether or not associated episodic details were successfully encoded. The analysis included four trial events: all trials, encoding trials with associated value ratings as a parametric modulator orthogonalized to the main encoding trial regressor, encoding 
VALUE NETWORK DURING ENCODING AND RETRIEVAL

trials with subsequent product attribute memory test responses as a parametric modulator orthogonalized to the main encoding trial regressor, and the interaction of encoding value rating and attribute memory test response. Trials were modeled by time of onset and participant response time for duration. The primary contrasts of interest examined the main effect of encoding trials associated with successful subsequent memory and the interaction of parametric value rating and product attribute memory test regressors. To examine valuation and episodic memory interactions at the retrieval phase, a parallel GLM analysis assessed activation related to successful episodic retrieval, with trials modeled by onset time and full trial duration ( 4 sec). The retrieval-oriented analysis design followed the same structure as in the encoding phase, with the purpose of identifying the neural correlates of value-retrieval trials associated with successful memory and the interaction of parametric retrieval value rating and product attribute memory test regressors. These neural analyses were aimed at determining if episodic memory and valuation mechanisms interacted, and if so, whether interactions indicated cooperation (i.e., enhanced engagement in valuation mechanisms with episodic processing) or competition (i.e., reduced engagement in valuation mechanisms with episodic processing).

\section{Results}

\subsection{Behavioral results}

\subsubsection{Successful retrieval of value ratings}

First, we confirmed that value ratings at encoding scaled linearly with the preset valence condition $\left(F_{(1.32,25.07)}=513.06, p<.001, \eta_{p}^{2}=.96\right)$. Post-hoc pairwise comparisons verified significant differences between rating conditions, such that ratings were higher for product comments in the positive $(M=3.56, S E M=0.06)$ compared to negative $(M=1.48, S E M=0.04$; $\left.t_{(19)}=-24.01, p_{h o l m}<.001, d=-5.37\right)$ valence condition. Product ratings were also confirmed to 
VALUE NETWORK DURING ENCODING AND RETRIEVAL

be less positive for products in the negative compared to neutral $(M=2.92, S E M=0.05 ; t(19)=-$

24.21, $\left.p_{\text {holm }}<.001, d=-5.41\right)$ condition and more positive for the positive compared to neutral

$(t(19)=-13.59$, pholm $<.001, d=-3.04)$ condition.

We further confirmed that value-related information presented at encoding was remembered during retrieval, as product-specific value ratings from the encoding phase strongly predicted value ratings from the retrieval phase $\left(F_{(1,2881)}=786.91, b=1.60, S E_{b}=0.06, p<.001, \eta_{p}^{2}=.22\right.$;

Fig. 4). The direction and strength of the relationship between value ratings in the two phases indicates that higher value at encoding corresponded to higher value at later retrieval. We did not find an effect of the number of encoding experiences $\left(F_{(1,2881)}=0.63, b=0.03, S E_{b}=0.03, p=\right.$ $\left..43, \eta_{p}^{2}<.001\right)$, indicating that the valence of value ratings at retrieval was not systematically different for products that were presented with one or two consumer reviews during encoding.

Additional analyses confirmed that the two consumer reviews presented with two-experience products during encoding both contributed to later retrieval ratings (see Supplementary Material, Section I). Results confirmed that value information was integrated across product-specific encoding trials; however, the first product comment appeared to have more influence than the second on subsequently retrieved value ratings. Together, these results signify the successful retrieval of value-related information presented during encoding across encoding experience conditions. Critically, previous research confirms that differences in neural recruitment due to experienced task demands can be observed in the absence of behavioral differences (e.g., Cabeza et al., 2004; Cappell, Gmeindl, \& Reuter-Lorenz, 2011; Nagel et al., 2013). As such, we included the number of encoding experiences as a factor in our fMRI analyses to test for differences in neural recruitment when value retrieval required accessing memory for one encoding event versus accessing and integrating two encoding events. 
VALUE NETWORK DURING ENCODING AND RETRIEVAL

\section{Figure 4}

Correspondence between value ratings across value encoding and retrieval

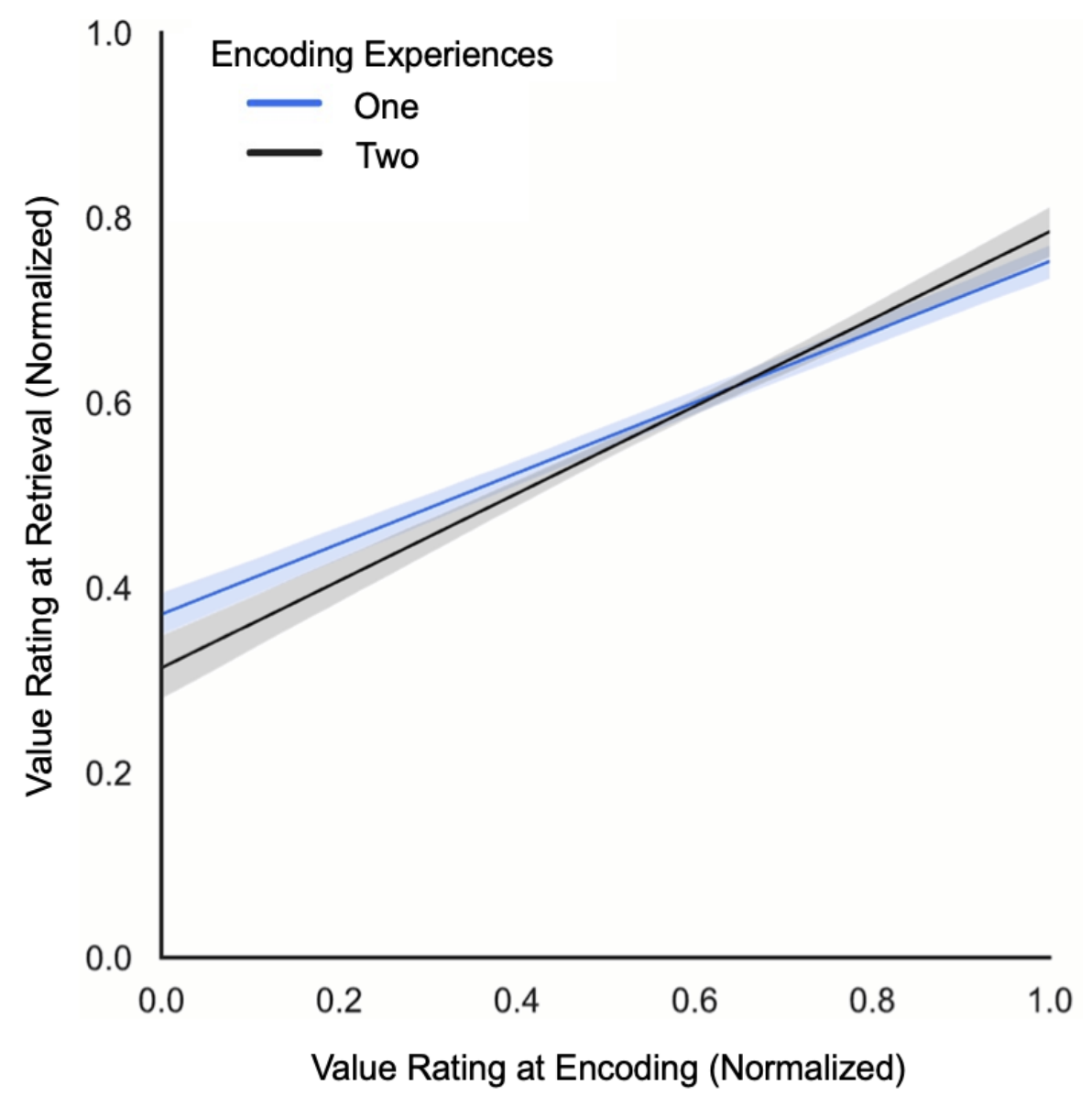

Note. Normalized value ratings during value retrieval were found to scale alongside value encoding ratings (averaged for products with two consumer reviews), regardless of the number of encoding experiences. For visual comparison purposes, discrete value encoding ratings (Xaxis) and continuous value retrieval ratings ( $\mathrm{Y}$-axis) were normalized to a $0-1$ scale, representing negative (0) to positive (1) value ratings. Shading represents $95 \%$ confidence intervals for each regression line. 
VALUE NETWORK DURING ENCODING AND RETRIEVAL

\subsubsection{Relationship between value retrieval and episodic memory performance}

We next examined performance on the product attribute memory task, confirming successful memory retrieval with an average corrected recognition score of $46 \%(S E M=0.04 ; t(19)=11.41$, $p<.001, d=5.24)$, corresponding to a hit rate of $79 \%$ and a false alarm rate of $34 \%$. Toward our goal of examining interactions between value- and memory-related processing, results of a triallevel linear mixed-effects model examining the relationship between value-retrieval accuracy and attribute memory showed that value-retrieval accuracy $(M=0.80, S E M=0.01)$ predicted better subsequent memory for product attributes $\left(F_{(1,2876.53)}=22.42, b=0.17, S E_{b}=0.04, p<\right.$ $\left..001, \eta_{p}^{2}=.13\right)$. Thus, our behavioral results indicate a correspondence between memory for value-related information and episodic details.

\subsection{Neuroimaging results}

\subsubsection{Distinct and common neural correlates of value encoding and retrieval}

3.2.1.1. Identification of phase-distinct versus common valuation regions. To address our primary study goal, we identified BOLD responses associated with value ratings at value encoding and retrieval. FDR-corrected value-encoding and value-retrieval activation maps were then compared to identify phase-distinct and -common valuation mechanisms.

In line with our first hypothesis, subtractions of the FDR-corrected value-weighted encoding and retrieval contrast images (i.e., Encoding > Retrieval) identified non-overlapping clusters of primary fronto-striatal and secondary fronto-parietal valuation network activity that scaled significantly with value ratings at each phase (Fig. 5a; Table 1 for cluster information). Focal clusters of activity were distinctly associated with value at encoding within the striatal left anterior caudate nucleus and right caudal body, along with the left superior parietal lobule (FDRcorrected threshold of $p<.0001)$. Notably, while canonical fronto-striatal reward and salience 
VALUE NETWORK DURING ENCODING AND RETRIEVAL

regions were recruited during our value-encoding task (Supplementary Material, Section III,

Table S1), activity within striatal and posterior parietal areas was most robustly connected to value rating behavior at encoding.

\section{Figure 5}

Phase-distinct and common neural activity related to value ratings

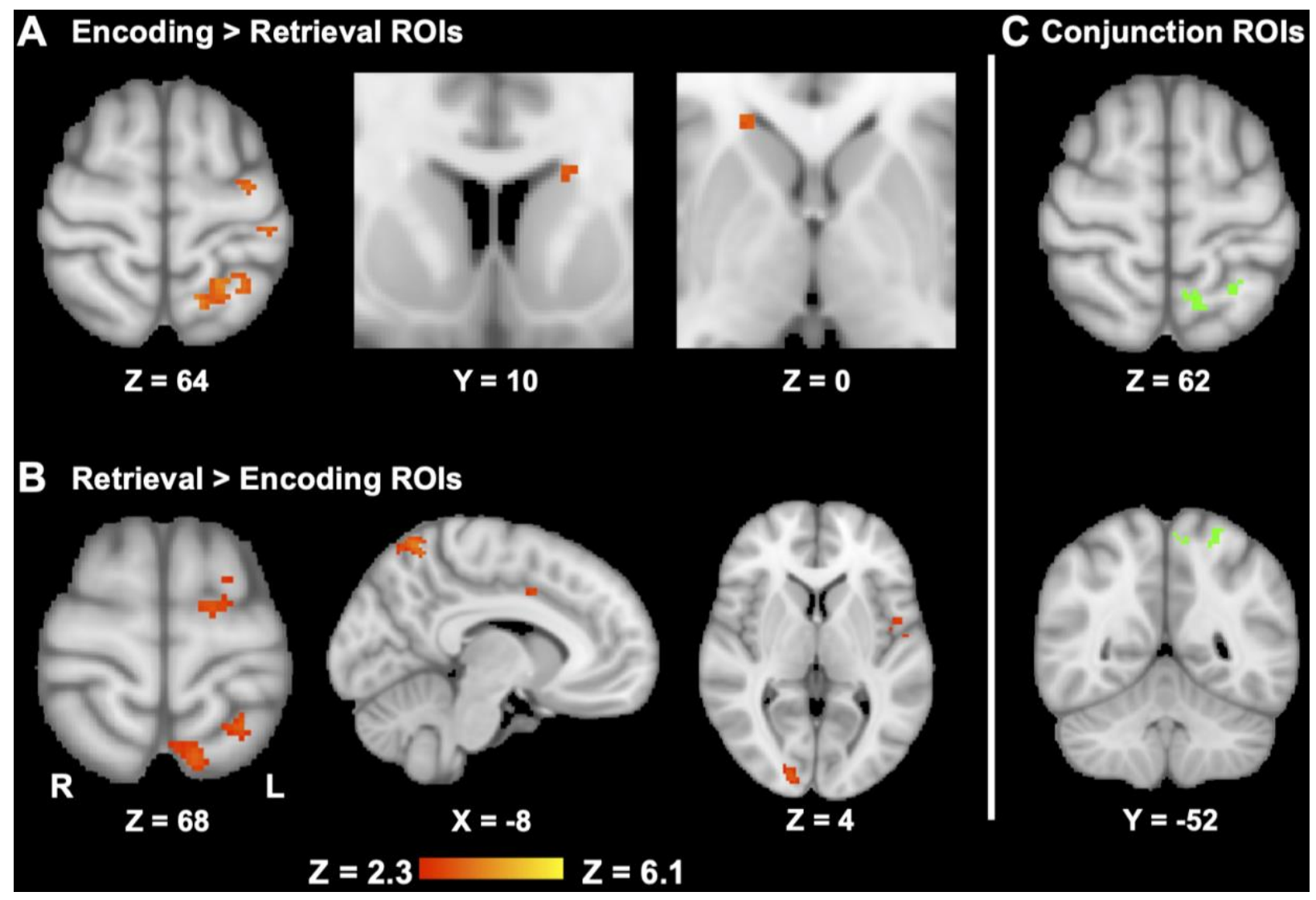

Note. (A) Greater overall recruitment of fronto-striatal valuation and fronto-parietal control network areas was associated with encoding and integrating value information, with areas of the bilateral striatal caudate nucleus and left superior parietal lobule surviving FDR-correction (voxel-wise threshold of $q=.05$ ). (B) While retrieving value information from recent memory, 
VALUE NETWORK DURING ENCODING AND RETRIEVAL

robust neural recruitment was primarily observed in salience and fronto-parietal control network areas, including superior frontal, insular, dorsal anterior cingulate, precuneal, and superior parietal clusters. (C) Recruitment of fronto-parietal and sensory processing regions was associated with value processing across valuation phases, with clusters in the left superior parietal lobule, precuneus, and right occipital fusiform areas surviving FDR-correction. Image slices are presented in radiological orientation with MNI slice coordinates. 
VALUE NETWORK DURING ENCODING AND RETRIEVAL

Table 1 Value-related activity distinct or common to valuation phases (FDR-corrected)

\begin{tabular}{|c|c|c|c|c|c|}
\hline & \multirow{2}{*}{$\begin{array}{l}\text { Cluster } \\
\text { Extent }\end{array}$} & \multicolumn{3}{|c|}{ MNI } & \multirow{2}{*}{$\begin{array}{l}\text { Peak } \\
\text { Z-stat }\end{array}$} \\
\hline Region & & $\mathrm{X}$ & $\mathrm{Y}$ & $\mathrm{Z}$ & \\
\hline \multicolumn{6}{|l|}{ Value Encoding (Encoding > Retrieval) } \\
\hline L Superior Parietal Lobule & 126 & -22 & -50 & 64 & 4.44 \\
\hline R Temporal Occipital Fusiform Cortex / & 52 & 26 & -56 & -16 & 4.27 \\
\hline \multicolumn{6}{|l|}{ R Occipital Fusiform Gyrus / Lingual Gyrus } \\
\hline L Postcentral Gyrus & 42 & -42 & -28 & 60 & 4.55 \\
\hline L Occipital Fusiform Gyrus & 29 & -38 & -70 & -12 & 4.01 \\
\hline L Inferior Occipital Cortex & 28 & -36 & -80 & 4 & 4.20 \\
\hline R Inferior Occipital Cortex & 22 & 50 & -72 & -10 & 4.00 \\
\hline Cerebellum & 13 & 18 & -66 & -18 & 3.93 \\
\hline L Superior Occipital Cortex & 8 & -22 & -72 & 38 & 3.92 \\
\hline L Precentral Gyrus & 8 & -32 & -12 & 64 & 4.19 \\
\hline R Caudate Nucleus (Anterior) & 7 & 20 & 28 & 0 & 3.96 \\
\hline L Caudate Nucleus (Body) & 5 & -15 & 9 & 20 & 3.94 \\
\hline \multicolumn{6}{|l|}{ Value Retrieval (Retrieval > Encoding) } \\
\hline Lingual Gyrus / R Occipital Fusiform Gyrus / & 1457 & 6 & -78 & -8 & 6.06 \\
\hline \multicolumn{6}{|l|}{ R Inferior Occipital Cortex } \\
\hline Precuneus / L Superior Occipital Cortex / & 159 & -10 & -60 & 64 & 4.41 \\
\hline \multicolumn{6}{|l|}{ L Superior Parietal Lobule } \\
\hline L Superior Parietal Lobule & 36 & -26 & -50 & 68 & 3.88 \\
\hline L Superior Frontal Gyrus & 29 & -16 & -8 & 70 & 3.88 \\
\hline Anterior Cingulate Cortex (Dorsal) & 10 & -8 & 4 & 38 & 3.65 \\
\hline $\begin{array}{l}\text { L Insular Cortex / Central Opercular Cortex / } \\
\text { Planum Polare }\end{array}$ & 6 & -50 & 2 & 4 & 3.57 \\
\hline \multicolumn{6}{|l|}{ Across Phases (Encoding x Retrieval) } \\
\hline R Occipital Fusiform Gyrus & 67 & 20 & -77 & -15 & - \\
\hline Precuneus / L Superior Parietal Lobule / & 39 & -11 & -56 & 64 & - \\
\hline \multicolumn{6}{|l|}{ L Superior Occipital Cortex } \\
\hline L Superior Parietal Lobule & 30 & -26 & -50 & 64 & - \\
\hline
\end{tabular}

Note. Cluster peak coordinates represent local maxima and are presented in MNI space. Reported activation clusters survived voxel-wise FDR multiple comparison correction (threshold of $q=.05$ ) and are greater than 4 voxels in size. Anatomical labels are presented for the primary regions represented within each cluster based on Harvard-Oxford cortical and subcortical structural atlas labels. $\mathrm{L}=$ left lateralized; $\mathrm{R}=$ right lateralized. 
VALUE NETWORK DURING ENCODING AND RETRIEVAL

Also in line with our first hypothesis, the regions identified during value retrieval (i.e., Retrieval > Encoding) matched with secondary valuation and memory mechanisms (frontoparietal control and salience networks), rather than the canonical fronto-striatal valuation network. During value retrieval, distinctive activity was identified within fronto-parietal and salience network regions, including clusters within the left insular cortex, left superior frontal gyrus, and dorsal anterior cingulate cortex (Fig. 5b; FDR-corrected threshold of $p<.0003$ ). Additional clusters spanned posterior parietal regions, encompassing the left superior parietal lobule and superior precuneus cortex (Table 1 for cluster information).

To determine areas recruited during both value encoding and retrieval, we utilized a conjunction comparison of value-weighted activity across valuation phases (i.e., Encoding x Retrieval). In comparing FDR-corrected cluster masks across phases, significant activation overlap was identified within precuneal, left superior parietal, and right occipital fusiform clusters adjacent to those identified in the phase-subtraction contrasts (Fig. 5c; Table 1).

As described in the Methods (Section 2.6.1.3), comparable neural correlates of valuation across phases were identified in additional analyses of value-related activity for products with one encoding experience (i.e., representing value-related activity without information integration; Supplementary Material, Section IV, Fig. S1, Table S2).

3.2.1.2. Direct comparison of valuation ROI activity across valuation phases. ROIs from the value network contrast analysis were used to test for significant differences in value-related activation by encoding and retrieval phase (for full details of ROI cluster information and associated analyses, see Supplementary Material, Section VI). ROIs resulting from the Encoding $>$ Retrieval contrast mask were in the right anterior caudate, left caudate, and left superior parietal areas. RM-ANOVA results indicated that value-related signal did not differ for encoding 
VALUE NETWORK DURING ENCODING AND RETRIEVAL

and retrieval phases across these ROIs $\left(F_{(1,19)}=0.74, p=.40, \eta_{p}^{2}=.04\right.$; Fig. 6a), and there was no significant interaction between valuation phase and ROIs $\left(F_{(2,38)}=0.44, p=.65, \eta_{p}^{2}=.02\right)$.

ROIs resulting from the Retrieval > Encoding contrast mask were in the left insula, left superior frontal gyrus, dorsal anterior cingulate cortex, left superior parietal lobule, and superior precuneus. RM-ANOVA results for value-related activity within these ROIs yielded no significant interactions $\left(F_{(2.29,43.42)}=0.60, p=.58, \eta_{p}{ }^{2}=.03\right)$, but did reveal a main effect of valuation phase $\left(F_{(1,19)}=5.77, p=.03, \eta_{p}^{2}=.23 ;\right.$ Fig. $\left.6 \mathrm{~b}\right)$. Post-hoc paired-samples t-tests revealed medium significant effects of valuation phase in three ROIs - the left insula $\left(t_{(19)}=-\right.$ $2.75, p=.01, d=-.62)$, precuneus $\left(t_{(19)}=-2.75, p=.01, d=-.61\right)$, and dorsal anterior cingulate gyrus $\left(t_{(19)}=-2.26, p=.04, d=-.51\right)$. The direction of the test statistics indicated significantly greater activity within these ROIs during value retrieval compared to value encoding. Phase differences in activation for the left superior frontal gyrus ROI did not reach significance $\left(t_{(19)}=-\right.$ $1.89, p=.07, d=-.42)$

ROIs resulting from the Encoding $\mathrm{x}$ Retrieval conjunction mask were in the precuneus and left superior parietal cortex. Consistent with expectation, RM-ANOVA results for value-related activity within these ROIs showed no significant differences between phases $\left(F_{(1,19)}=0.16, p=\right.$ $.70, \eta_{p}^{2}=.01$; Fig. $\left.6 \mathrm{c}\right)$, nor evidence of an interactive effect of ROI and valuation phase $\left(F_{(1,19)}=\right.$ $\left.3.56, p=.08, \eta_{p}^{2}=.16\right)$. 


\section{VALUE NETWORK DURING ENCODING AND RETRIEVAL}

\section{Figure 6}

Comparison of ROI cluster activity levels across valuation phases

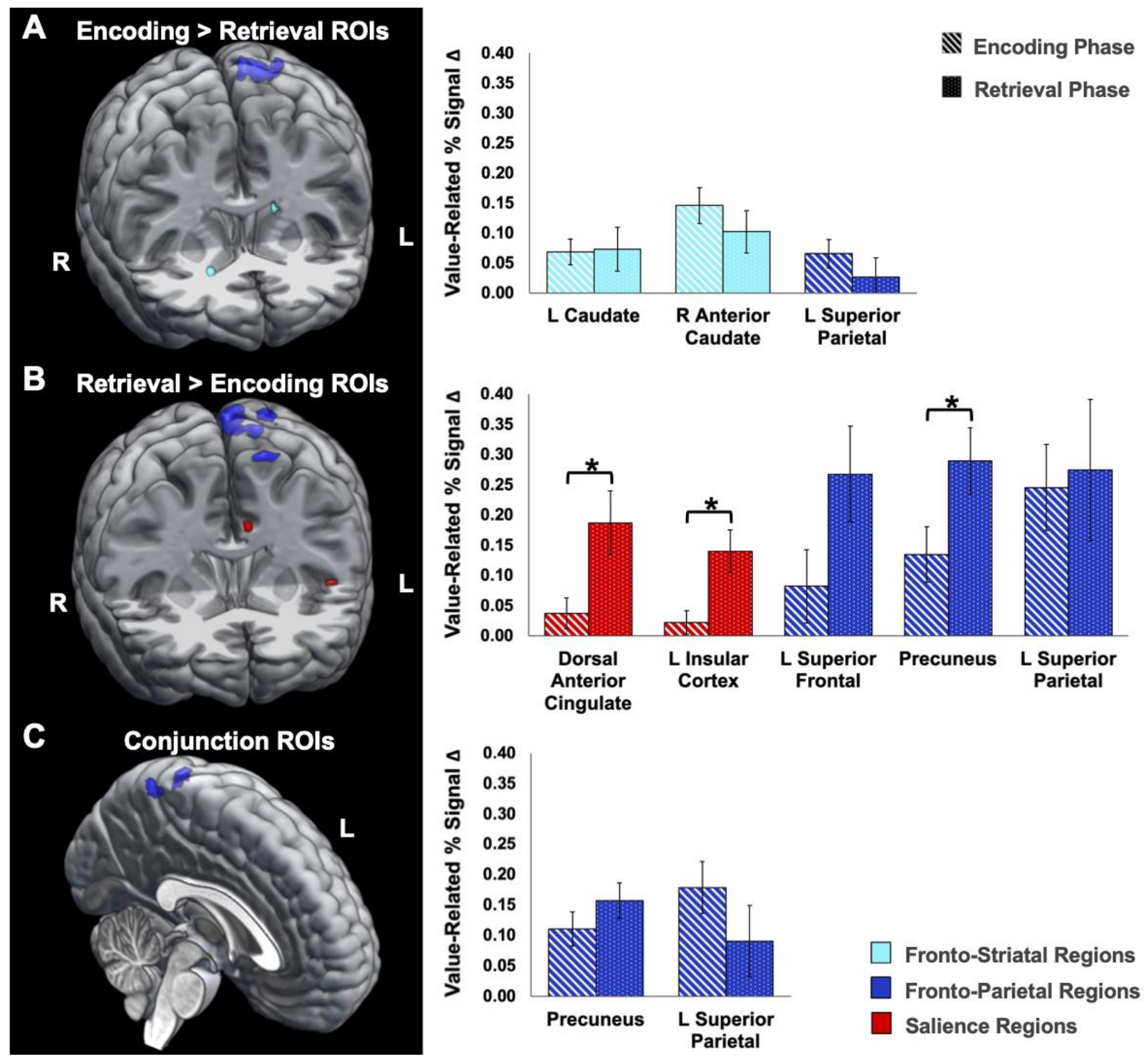

Note. (A) Similar value-related activation levels were observed across valuation phases within the caudal (light blue) and superior parietal (dark blue) clusters identified from the value encoding subtraction contrast, reflecting dependence on striatal and posterior parietal areas for 
VALUE NETWORK DURING ENCODING AND RETRIEVAL

value processing whether the value details were present or retrieved from recent memory. (B)

During the value-retrieval phase, however, significantly greater activity was observed for the dorsal anterior cingulate gyrus (red; $t_{(19)}=-2.26, p=.04, d=-.51$ ), left lateralized insular cortex $\left(\right.$ red; $\left.t_{(19)}=-2.75, p=.01, d=-.62\right)$, and precuneus (dark blue; $\left.t_{(19)}=-2.75, p=.01, d=-.61\right)$ in comparison to activity at value encoding. (C) Posterior parietal ROIs (dark blue) identified from a conjunction analysis represent regions recruited for value processing across phases and therefore were not expected to have significant activation differences. Error bars represent standard error of the mean; asterisks $(*)$ indicate significant differences in activity between phases at $p<.05$.

Together, the tests of ROI activity suggest reliance on posterior parietal and fronto-striatal valuation regions for both encoding and retrieval of value information, with significantly greater recruitment of select parietal control and salience regions during the retrieval of value. Further, consistently high recruitment of adjacent clusters within the left superior parietal lobule and precuneus across phases suggests a strong reliance on the posterior parietal cortex across value encoding and retrieval.

\subsubsection{Relationship between valuation and episodic memory systems}

Toward our secondary goal, we examined brain activation corresponding to episodic memory for product attributes relative to the neural mechanisms of value. GLM analyses examined interactions between regressors representing valuation and successful episodic memory for product attributes. In the encoding and retrieval phases, we observed neural correlates of successful subsequent memory for product attributes across regions commonly associated with episodic memory, but associated brain activation did not survive FDR correction (Supplementary 
VALUE NETWORK DURING ENCODING AND RETRIEVAL

Material, Section VII). Most notably, interaction contrasts between valuation and subsequent attribute memory did not yield any significant clusters during either phase. These analyses uniformly support independence, rather than interaction, between neural correlates of valuation and episodic memory.

\section{Discussion}

Everyday decision making requires efficient assessment of available options, which often involves active evaluation and retrieval of previous experiences. To determine how encoding and retrieval processes affect the composition of the value network, we used a novel memorydependent valuation paradigm and fMRI to compare behavior and neural processing during encoding versus retrieval of value. Further, to clarify the nature of the relationship between valuation and memory systems, we examined evidence for interactions between the neural mechanisms of valuation and episodic memory processes. Consistent with our first hypothesis and prior studies of valuation, our whole brain analysis of value-related activation revealed significant engagement of striatal regions in the canonical valuation network during value encoding. Diverging slightly from our prediction, however, direct comparison of valuationassociated activity in functionally determined ROIs indicated similar fronto-striatal network engagement during value encoding and retrieval. Our results also revealed robust value-related activation in posterior parietal regions across phases, while the value-retrieval phase was associated with significantly greater engagement of salience and parietal regions previously implicated in valuation. Finally, our examination of valuation and episodic memory interactions largely supported independence of these neural systems in task contexts where value judgments are based on episodic encoding of value and associates are not salient at value retrieval. 
VALUE NETWORK DURING ENCODING AND RETRIEVAL

\subsection{Distinct and common neural correlates of value encoding and retrieval}

This study aimed to identify distinctive and common neural correlates of valuation during encoding and retrieval. Our findings showed that neural engagement of striatal and posterior parietal regions was similar across valuation phases, while highlighting distinctive reliance on the precuneus and salience network regions during value retrieval. In accordance with our first prediction, results from the value-encoding phase indicated recruitment of key fronto-striatal regions commonly implicated in valuation paradigms (see Hsu \& Goh, 2016; Kable \& Glimcher, 2009; Levy \& Glimcher, 2012; Rangel, Camerer, \& Montague, 2008; Rushworth et al., 2011 for review), particularly the striatal caudate nucleus, in addition to the left superior parietal lobule.

Notably, however, a direct comparison of value-related activity across encoding and retrieval from functionally determined value-encoding ROIs yielded relative similarity in activation across phases. This result indicates a similar reliance on striatal and posterior parietal regions during encoding and retrieval of value. In addition to fronto-parietal control areas, salience network regions including the anterior insula and dorsal anterior cingulate were uniquely associated with value retrieval. Direct comparison of value-related BOLD activity within functionally determined ROIs across phases further confirmed significantly greater value-related engagement of the dorsal anterior cingulate, left insular, and precuneal cortices during value retrieval in comparison to value encoding.

Recruitment of fronto-striatal circuitry during the assessment and integration of value based on presented product information is consistent with previously identified mechanisms of value encoding and updating from reinforcement learning and one-shot decision tasks (Bartra, McGuire, \& Kable, 2013; Delgado, 2007; Foerde \& Shohamy, 2011; Jocham et al., 2014; Kable \& Glimcher, 2007; Kable \& Glimcher, 2009; Knutson et al., 2005; Koscik et al., 2020; Levy et 
VALUE NETWORK DURING ENCODING AND RETRIEVAL

al., 2010; McClure et al., 2004; Peters \& Büchel, 2009; Shenhav, Barrett, \& Bar, 2013; Strait,

Sleezer, \& Hayden, 2015). In particular, our analyses highlighted the role of the caudate nucleus in valuation during our task, which involved assigning valence weightings to consumer products based on "consumer reviews" that were present (encoding) or called from memory (retrieval). The specific location of observed value-related activation during our task was within the anterior caudate (head) and posterior caudate (body), rather than the more typically implicated nucleus accumbens. While the ventral striatum has a well-established reward-processing role in representing and updating stimulus value (Daw et al., 2011; Haber, 2011; Knutson, Adams, Fong, \& Hommer, 2001; Levy et al., 2010; Morris et al., 2016; O’Doherty et al., 2004; Peters \& Büchel, 2010; Strait, Sleezer, \& Hayden, 2015), the anterior and posterior caudal regions have more recently been connected to evaluating action outcomes and exerting cognitive control, respectively, toward value-mediated action selection (Choi, Shin, \& Kim, 2020; Pauli, O'Reilly, Yarkoni, \& Wager, 2016; Tricomi \& Lempert, 2015; Watson, van Wingen, \& de Wit, 2018).

Our study also yielded robust value-related activation of posterior parietal regions, while the ventromedial prefrontal cortex was not as robustly activated during our experiment. Taskspecific effects may explain the relative robustness of fronto-striatal versus fronto-parietal region engagement during valuation. The ventromedial prefrontal cortex has been specifically implicated in deliberation of value differences toward value maximization - particularly in evaluating expected value from choice outcomes or with self-relevant reward or punishment outcomes (Camille, Griffiths, Vo, Fellows, \& Kable, 2011; Grabenhorst \& Rolls, 2011; Hunt et al., 2012; Kim, Shimojo, \& O’Doherty, 2011; Kim \& Johnson, 2015; Levy \& Glimcher, 2012; Lin, Horner, \& Burgess, 2016; Nicolle et al., 2012; Roy, Shohamy, \& Wager, 2012; Wunderlich, Rangel, \& O’Doherty, 2009). 
VALUE NETWORK DURING ENCODING AND RETRIEVAL

While our task did include monetary incentives designed to elicit self-relevant value responses, it did not include trial-wise choices or reinforcements, which may have reduced the immediate self-relevance of our stimuli compared with those in previous studies. Beyond selfrelevant values, the observation of robust value-related activation in the superior parietal lobule and larger fronto-parietal network is in line with previous connections to choice action planning, trial-by-trial updating, and integration of bottom-up sensory information with top-down task goals (Andersen \& Buneo, 2002; Cohen et al., 2014; Cole, Repovs, \& Anticevic, 2014; Park \& Kayser, 2019; Ptak, 2011; Tosoni et al., 2014; Yeo et al., 2011).

Furthermore, fitting with value encoding in our task, value comparisons have been associated with superior parietal lobule engagement when value choices are made under restricted time limits, with the ventromedial prefrontal cortex keeping track of overall value (Domenech, Redouté, Koechlin, \& Dreher, 2018; Jocham et al., 2014). In a recent study, Koscik and colleagues (2020) used a simple 50/50 chance gambling task to examine specific components of choice valuation. Their results suggested that the ventral striatum extracts value information and compares choice options, while the posterior parietal cortex extracts value magnitude information with no need for a mediating role of the ventromedial prefrontal cortex. Future research that compares choices with more immediate self-relevance versus more abstract valuation will help clarify the unique roles that fronto-striatal and fronto-parietal regions play in valuation and value-based decision making.

Our findings of retrieval-distinct neural correlates of value include significantly greater recruitment of dorsal anterior cingulate and insular regions during value retrieval. These results suggest that salience network regions play a stronger role in valuation when value judgments must be made from recently encoded representations in episodic memory. Salience network 
VALUE NETWORK DURING ENCODING AND RETRIEVAL

regions contribute to value processing by selecting, integrating, and utilizing goal-relevant sensory information (Dosenbach et al., 2008; Ham et al., 2013; Hélie, Shamloo, Novak, \& Foti, 2017; Kahnt, Park, Haynes, \& Tobler, 2014; Koscik et al., 2020; Krebs, Boehler, Roberts, Song, \& Woldorff, 2012; Litt, Plassmann, Shiv, \& Rangel, 2011; Menon \& Uddin, 2010; Seeley et al., 2007). The anterior insula and dorsal anterior cingulate have also been specifically implicated in assessing choice uncertainty and utilizing available value representations to resolve ambiguity (Lamichhane \& Dhamala, 2015; Loued-Khenissi, Pfeuffer, Einhäuser, Preuschoff, 2020;

Rushworth \& Behrens, 2008; Stöttinger, Aichhorn, Anderson, \& Danckert, 2018). Our results are congruent with these prior studies, suggesting that salience-based components of value representations are less vulnerable to degradation from encoding to retrieval, and make greater contributions to valuation as dependence on memory causes uncertainty about value.

We also observed greater engagement of select fronto-parietal control regions during value retrieval, particularly in the precuneus. Posterior parietal regions support planning and initiating value-based behaviors (Andersen \& Buneo, 2002; Clithero, Carter, \& Huettel, 2009; Domenech et al., 2018; Tosoni et al., 2014). Of particular relevance to value retrieval, prior studies have implicated the precuneus in accessing stored mental representations of value and regenerating associated context (Johnson, Mitchell, Raye, D’Esposito, \& Johnson, 2007; Lundstrom, Ingvar, \& Petersson, 2005; Zhang \& Li, 2012). Thus, collectively, our findings add to the growing literature that suggests salience network and posterior parietal regions play a critical role in the retrieval of value representations from memory.

While select subregions of the posterior parietal cortex may be recruited to a greater extent during the value retrieval phase in our task, our results support the conclusion that posterior parietal regions are robustly associated with value rating behavior whether value-based 
VALUE NETWORK DURING ENCODING AND RETRIEVAL

information is being encoded or retrieved from memory. Recruitment of posterior parietal regions across value encoding and retrieval is consistent with research showing these regions support the accumulation of choice-relevant information and the transformation of integrated representations into behavioral responses (Andersen \& Buneo, 2002; Clithero, Carter, \& Huettel, 2009; Domenech et al., 2018; Kable \& Glimcher, 2009; Park \& Kayser, 2019). Further, superior subregions of the posterior parietal cortex have been associated with goal-directed valuation beyond bottom-up salience-driven responses (Kahnt et al., 2014; Koscik et al., 2020; Uncapher \& Wagner, 2009). In the current study, the posterior parietal regions were paramount to both actively encoding and integrating value information while product comments were present, and may represent accumulated value information to initiate a rating response.

\subsection{Relationship between valuation and episodic memory systems}

Our secondary study goal was to examine potential interactions between valuation and episodic memory systems. Behaviorally, we found an association between retrieval of value and attribute details, indicating a correspondence in memory for product values and those products' features. Our neural data, however, did not yield evidence of an interaction between valuation and memory processing; nor did we observe any effects of the number of encoding experiences on value-related brain activation. In accordance with our second hypothesis, these results indicate independence of neural mechanisms of value and memory-related processing.

This conclusion conflicts with previous reports suggesting competition between valuation and memory systems, but differing results are likely due to task conditions. For example, Wimmer and colleagues (2014) previously found that incidental encoding of value-irrelevant episodic details (i.e., overlaid object images) during a reinforcement-learning task was associated with decreased influence of recent reward outcomes on subsequent choice. In contrast, product 
VALUE NETWORK DURING ENCODING AND RETRIEVAL

values in the present experiment were based on limited prior experience, allowing for effective episodic encoding of value. In addition, episodic information encountered in our valuation task was relevant to information about the product value, as attribute details were embedded in our consumer review stimuli. Our results suggest that in such contexts, stimuli values and episodic associates can be learned in parallel - without competition for processing resources. Thus, our findings are more consistent with behavioral research showing a relationship between memory for associates of choice stimuli and value-based decision performance when choice values can be effectively encoded and retrieved via episodic memory (Duncan \& Shohamy, 2016; Murty et al., 2016).

Notably, results from these behavioral paradigms indicated facilitation of value-based decisions with better memory for choice associates. While our behavioral results are generally congruent with these studies, our functional brain imaging results indicate independent neural mechanisms of valuation and episodic memory. An important and distinctive feature of the present study's paradigm is that memory-associates (product attribute cue words) were not made salient during the valuation phases. Indeed, cue words were not present during value retrieval. In behavioral studies indicating cooperation between value and memory systems, stimuli previously paired with choice options were present in value-based decision context (Duncan \& Shohamy, 2016), or they were the choice stimulus itself (Murty et al., 2016). When combined with findings from the present study, extant research suggests that manipulating the salience of memory associates during value learning and value-based choice may impact the degree to which neural mechanisms of value and memory show cooperation or facilitation effects. 
VALUE NETWORK DURING ENCODING AND RETRIEVAL

\subsection{Concluding remarks}

Naturalistic decision making often involves encoding incoming value information and integrating that information with previous experiences to select the best choice option. Although previous studies have identified regions associated with valuation, we argue that typical valuation paradigms involve the encoding and integration of present value information more than strict retrieval-based valuation. The present study addressed this issue by dissociating valuation in distinct phases of memory using ecologically valid stimuli. By distinguishing between the neural correlates underlying value processing at different phases of memory, we advance understanding of the value network's composition during value encoding versus retrieval.

Further, the present study provides novel insights into the conditions under which neural mechanisms of value and episodic memory will interact. Considered alongside prior literature, our findings suggest that the number of interactions with choice stimuli and salience of memory associates during value-based choice likely determine the presence and nature of interactions between the neural mechanisms of valuation and episodic memory. These findings hold relevance for much of our everyday decision making, ranging from shopping to selecting between healthcare plan options, and demonstrate how recent exposures with product marketing may affect value-based processing. 
VALUE NETWORK DURING ENCODING AND RETRIEVAL

\section{Funding}

This research did not receive any specific grant from funding agencies in the public, commercial, or not-for-profit sectors. MH was supported by a Pre-Doctoral Fellowship on Physical, Cognitive \& Mental Health in Social Context from the National Institute on Aging (T32AG020499) and a Post-Doctoral Fellowship from the UF Substance Abuse Training Center in Public Health from the National Institute of Drug Abuse of the National Institutes of Health (T32DA035167). The content is solely the responsibility of the author(s) and does not necessarily represent the official views of the National Institutes of Health. A portion of this work was performed in the McKnight Brain Institute at the National High Magnetic Field Laboratory’s AMRIS Facility, which is supported by National Science Foundation Cooperative Agreement No. DMR-1644779 and the State of Florida.

\section{CRediT authorship contribution statement}

Lindsay B. Conner: Methodology, Software, Validation, Formal analysis, Investigation, Data curation, Visualization, Writing - original draft, Writing - review \& editing

Marilyn Horta: Methodology, Data curation, Software, Validation, Writing - review \& editing Natalie C. Ebner: Conceptualization, Supervision, Project administration, Funding acquisition, Resources, Writing - review \& editing

Nichole R. Lighthall: Conceptualization, Methodology, Supervision, Project administration, Resources, Funding acquisition, Writing - review \& editing

\section{Declaration of competing interest}

The authors declare no competing financial interests. 
VALUE NETWORK DURING ENCODING AND RETRIEVAL

\section{Acknowledgements}

The authors gratefully acknowledge the contributions of Ian Dalton, Ian Frazier, Desiree Lussier, and Eliany Perez for their assistance with protocol development, participant recruitment, data collection, and data management. We also gratefully acknowledge the contributions of Samuel Naranjo Rincón and Yerika Germosen toward quality control and data analysis procedures. We further thank the staff of the McKnight Brain Institute of the University of Florida for their assistance with MRI data collection and the participants for their time. 
VALUE NETWORK DURING ENCODING AND RETRIEVAL

\section{References}

Andersen, R. A. \& Buneo, C. A. (2002). Intentional maps in posterior parietal cortex. Annual Review of Neuroscience, 25(1), 189-220. doi:10.1146/annurev.neuro.25.112701.142922

Bartra, O., McGuire, J. T., \& Kable, J. W. (2013). The valuation system: A coordinate-based meta-analysis of BOLD fMRI experiments examining neural correlates of subjective value. NeuroImage, 76, 412-427. doi:10.1016/j.neuroimage.2013.02.063

Bhui, R. (2018). Case-based decision neuroscience: Economic judgment by similarity. In R. W. Morris, A. M. Bornstein, \& A. Shenhav (Eds.), Goal-directed decision making: Computations and neural circuits (pp. 67-103). Elsevier Academic Press. doi:10.1016/b978-0-12-812098-9.00004-8

Biswal, B. B., Mennes, M., Zuo, X. N., Gohel, S., Kelly, C., Smith, S. M., .. \& Castellanos, F. X. (2010). Toward discovery science of human brain function. Proc. Natl. Acad. Sci. USA, 107(10), 4734-4739.

Bornstein, A. M., Khaw, M. W., Shohamy, D., \& Daw, N. D. (2017). Reminders of past choices bias decisions for reward in humans. Nature Communications, 8, 15958. doi:10.1038/ncomms 15958

Bornstein, A. M. \& Norman, K. A. (2017). Reinstated episodic context guides sampling-based decisions for reward. Nature Neuroscience, 20(7), 997-1003.

Brosch, T. \& Sander, D. (2013). Neurocognitive mechanisms underlying value-based decisionmaking: From core values to economic value. Frontiers in Human Neuroscience, 7, 398. doi:10.3389/fnhum.2013.00398

Cabeza, R., Prince, S. E., Daselaar, S. M., Greenberg, D. L., Budde, M., Dolcos, F., LaBar, K. S., \& Rubin, D. C. (2004). Brain activity during episodic retrieval of autobiographical and 


\section{VALUE NETWORK DURING ENCODING AND RETRIEVAL}

laboratory events: an fMRI study using a novel photo paradigm. Journal of Cognitive Neuroscience, 16(9), 1583-1594. doi:10.1162/0898929042568578

Camille, N., Griffiths, C. A., Vo, K., Fellows, L. K., \& Kable, J. W. (2011). Ventromedial frontal lobe damage disrupts value maximization in humans. Journal of Neuroscience, 31(20), 7527-7532. doi:10.1523/jneurosci.6527-10.2011

Cappell, K. A., Gmeindl, L., \& Reuter-Lorenz, P. A. (2010). Age differences in prefrontal recruitment during verbal working memory maintenance depend on memory load. Cortex, 46(4), 462-473. doi:10.1016/j.cortex.2009.11.009

Carter, R. M., Meyer, J. R., \& Huettel, S. A. (2010). Functional neuroimaging of intertemporal choice models: a review. J. Neurosci. Psychol. Econ., 3, 27-45.

Choi, Y., Shin, E. Y., \& Kim, S. (2020). Spatiotemporal dissociation of fMRI activity in the caudate nucleus underlies human de novo motor skill learning. Proc. Natl. Acad. Sci. USA, 202003963. doi:10.1073/pnas.2003963117

Clithero, J. A., Carter, R. M., \& Huettel, S. A. (2009). Local pattern classification differentiates processes of economic valuation. NeuroImage, 45(4), 1329-1338. doi:10.1016/j.neuroimage.2008.12.074

Clithero, J. A. \& Rangel, A. (2014). Informatic parcellation of the network involved in the computation of subjective value. Social Cognitive and Affective Neuroscience, 9(9), 1289-1302.

Cohen, M., Rissman, J., Suthana, N. A., Castel, A. D., \& Knowlton, B. J. (2014). Value-based modulation of memory encoding involves strategic engagement of fronto-temporal semantic processing regions. Cognitive, Affective \& Behavioral Neuroscience, 14, 578592. doi:10.3758/s13415-014-0275-X 
VALUE NETWORK DURING ENCODING AND RETRIEVAL

Cole, M. W., Repovs, G., \& Anticevic, A. (2014). The frontoparietal control system: A central role in mental health. The Neuroscientist, 20(6), 652-664. doi: $10.1177 / 1073858414525995$

Daw, N. D., Gershman, S. J., Seymour, B., Dayan, P., \& Dolan, R. J. (2011). Model-based influences on humans' choices and striatal prediction errors. Neuron, 69(6), 1204-1215.

Delgado, M. R. (2007). Reward-related responses in the human striatum. Annals of the New York Academy of Sciences, 1104(1), 70-88. doi:10.1196/annals.1390.002

Dickerson, K. C., Li, J., \& Delgado, M. R. (2011). Parallel contributions of distinct human memory systems during probabilistic learning. NeuroImage, 55(1), 266-276.

Domenech, P., Redouté, J., Koechlin, E., \& Dreher, J.-C. (2018). The neuro-computational architecture of value-based selection in the human brain. Cerebral Cortex, 8, 585-601. doi:10.1093/cercor/bhw396

Dosenbach, N. U., Fair, D. A., Cohen, A. L., Schlaggar, B. L., \& Petersen, S. E. (2008). A dualnetworks architecture of top-down control. Trends in Cognitive Sciences, 12(3), 99-105.

Duncan, K. D. \& Shohamy, D. (2016). Memory states influence value-based decisions. Journal of Experimental Psychology, 145(11), 1420-1426. doi:10.1037/xge0000231

Enkavi, A. Z., Weber, B., Zweyer, I., Wagner, J., Elger, C. E., Weber, E. U., \& Johnson, E. J. (2017), Evidence for hippocampal dependence of value-based decisions. Scientific Reports, 7, 17738. doi:10.1038/s41598-017-18015-4

Foerde, K. \& Shohamy, D. (2011). The role of the basal ganglia in learning and memory: Insight from Parkinson's disease. Neurobiology of Learning and Memory, 96(4), 624-636. doi:10.1016/j.nlm.2011.08.006 
VALUE NETWORK DURING ENCODING AND RETRIEVAL

Friston, K. J., Holmes, A. P., Price, C. J., Büchel, C., Worsley, K. J. (1999). Multisubject FMRI studies and conjunction analyses. NeuroImage, 10, 385-396.

Genovese, C., Lazar, N., \& Nichols, T. (2002). Thresholding of statistical maps in functional neuroimaging using the false discovery rate. Neuroimage, 15, 870-878.

Gershman, S. J., Pesaran, B., \& Daw, N. D. (2009). Human reinforcement learning subdivides structured action spaces by learning effector-specific values. Journal of Neuroscience, 29(43), 13524-13531.

Gläscher, J., Adolphs, R., Damasio, H., Bechara, A., Rudrauf, D., Calamia, M., Paul, L. K., \& Tranel, D. (2012). Lesion mapping of cognitive control and value-based decision making in the prefrontal cortex. Proc. Natl. Acad. Sci. USA, 109, 14681-14686.

Gläscher, J., Daw, N., Dayan, P., \& O’Doherty, J. P. (2010). States versus rewards: Dissociable neural prediction error signals underlying model-based and model-free reinforcement learning. Neuron, 66(4), 585-595.

Gläscher, J., Hampton, A. N., \& O’Doherty, J. P. (2009). Determining a role for ventromedial prefrontal cortex in encoding action-based value signals during reward-related decision making. Cerebral Cortex, 19(2), 483-495.

Grabenhorst, F. \& Rolls, E. T. (2011). Value, pleasure and choice in the ventral prefrontal cortex. Trends Cogn. Sci., 15, 56-67. doi:10.1016/j.tics.2010.12.004

Haber, S. N. (2011). Neuroanatomy of reward: A view from the ventral striatum. In J. A. Gottfried (Ed.), Neurobiology of sensation and reward (pp. 235-261). CRC Press/Taylor \& Francis. doi:10.1201/b10776-15 
VALUE NETWORK DURING ENCODING AND RETRIEVAL

Ham, T., Leff, A., de Boissezon, X., Joffe, A., \& Sharp, D. J. (2013). Cognitive control and the salience network: An investigation of error processing and effective connectivity. The Journal of Neuroscience, 33(16), 7091-7098. doi:10.1523/JNEUROSCI.4692-12.2013

Hare, T., Camerer, C., \& Rangel, A. (2009). Self-control in decision-making involves modulation of the vmPFC valuation system. NeuroImage, 47, 646-648. doi:10.1016/s1053-8119(09)70776-1

Hélie, S., Shamloo, F., Novak, K., \& Foti, D. (2017). The roles of valuation and reward processing in cognitive function and psychiatric disorders. Annals of the New York Academy of Sciences, 1395, 33-48. doi:10.1111/nyas.13327

Hunt, L. T., Kolling, N., Soltani, A., Woolrich, M. W., Rushworth, M. F. S., \& Behrens, T. E. J. (2012). Mechanisms underlying cortical activity during value-guided choice. Nature Neuroscience, 15(3), 470-476. doi:10.1038/nn.3017

Hsu, C. W. \& Goh, J. O. (2016). Distinct and overlapping brain areas engaged during valuebased, mathematical, and emotional decision processing. Frontiers in Human Neuroscience, 10, 275. doi:10.3389/fnhum.2016.00275

Jimura, K., Chushak, M.S., \& Braver, T.S. (2013). Impulsivity and self-control during intertemporal decision making linked to the neural dynamics of reward value representation. Journal of Neuroscience, 33(1), 344-357.

Jocham, G., Furlong, P. M., Kröger, I. L., Kahn, M. C., Hunt, L. T., \& Behrens, T. E. (2014). Dissociable contributions of ventromedial prefrontal and posterior parietal cortex to value-guided choice. NeuroImage, 100, 498-506. doi:10.1016/j.neuroimage.2014.06.005

Johnson, M. R., Mitchell, K. J., Raye, C. L., D’Esposito, M., \& Johnson, M. K. (2007). A brief thought can modulate activity in extrastriate visual areas: Topdown effects of refreshing 
VALUE NETWORK DURING ENCODING AND RETRIEVAL

just-seen visual stimuli. Neuroimage, 37(1), 290-299.

doi:10.1016/j.neuroimage.2007.05.017

Jones, J. L., Esber, G. R., Mcdannald, M. A., Gruber, A. J., Hernandez, A., Mirenzi, A., \& Schoenbaum, G. (2012). Orbitofrontal cortex supports behavior and learning using inferred but not cached values. Science, 338(6109), 953-956.

doi:10.1126/science.1227489

Kable, J. W. \& Glimcher, P. W. (2007). The neural correlates of subjective value during intertemporal choice. Nature Neuroscience, 10(12), 1625-1633.

Kable, J. W. \& Glimcher, P. W. (2009). The neurobiology of decision: Consensus and controversy. Neuron, 63(6), 733-745.

Kahnt, T., Park, S. Q., Haynes, J.-D., \& Tobler, P. N. (2014). Disentangling neural representations of value and salience in the human brain. Proc. Natl. Acad. Sci. USA, 111(13), 5000-5005. doi:10.1073/pnas.1320189111

Kim, K. \& Johnson, M. K. (2015). Activity in ventromedial prefrontal cortex during self-related processing: Positive subjective value or personal significance? Social Cognitive and Affective Neuroscience, 10(4), 494-500. doi:10.1093/scan/nsu078

Kim, H., Shimojo, S., \& O’Doherty, J. P. (2011). Overlapping responses for the expectation of juice and money rewards in human ventromedial prefrontal cortex. Cerebral Cortex, 21(4), 769-776. doi:10.1093/cercor/bhq145

Knutson, B., Adams, C. M., Fong, G. W., \& Hommer, D. (2001). Anticipation of increasing monetary reward selectively recruits nucleus accumbens. J. Neurosci., 21, RC159. 
VALUE NETWORK DURING ENCODING AND RETRIEVAL

Knutson, B., Taylor, J., Kaufman, M., Peterson, R., \& Glover, G. (2005). Distributed neural representation of expected value. The Journal of Neuroscience, 25, 4806-4812. doi:10.1523/JNEUROSCI.0642-05.2005.

Koscik, T. R., Man, V., Jahn, A., Lee, C. H., \& Cunningham, W. A. (2020). Decomposing the neural pathways in a simple, value-based choice. NeuroImage, 214, 116764. doi:10.1016/j.neuroimage.2020.116764

Krebs, R. M., Boehler, C. N., Roberts, K. C., Song, A. W., \& Woldorff, M. G. (2012). The involvement of the dopaminergic midbrain and cortico-striatal-thalamic circuits in the integration of reward prospect and attentional task demands. Cerebral Cortex, 22(3), 607-615. doi:10.1093/cercor/bhr134

Kuhl, B. A., Shah, A. T., DuBrow, S., \& Wagner, A. D. (2010). Resistance to forgetting associated with hippocampus-mediated reactivation during new learning. Nature Neuroscience, 13(4), 501-506. doi:10.1038/nn.2498

Lamichhane, B. \& Dhamala, M. (2015). The salience network and its functional architecture in a perceptual decision: An effective connectivity study. Brain Connectivity, 5(6), 362-370. doi:10.1089/brain.2014.0282

Levy, D. J. \& Glimcher, P. W. (2012). The root of all value: A neural common currency for choice. Current Opinion in Neurobiology, 22(6), 1027-1038.

Levy, I., Snell, J., Nelson, A. J., Rustichini, A., \& Glimcher, P. W. (2010). Neural representation of subjective value under risk and ambiguity. Journal of Neurophysiology, 103(2), 10361047. doi:10.1152/jn.00853.2009

Lin, W., Horner, A. \& Burgess, N. (2016). Ventromedial prefrontal cortex, adding value to autobiographical memories. Scientific Reports, 6, 28630. doi:10.1038/srep28630 
VALUE NETWORK DURING ENCODING AND RETRIEVAL

Litt, A., Plassmann, H., Shiv, B., \& Rangel, A. (2011). Dissociating valuation and saliency signals during decision-making. Cerebral Cortex, 21(1), 95-102. doi:10.1093/cercor/bhq065

Liu, X., Hairston, J., Schrier, M., \& Fan, J. (2011). Common and distinct networks underlying reward valence and processing stages: A meta-analysis of functional neuroimaging studies. Neuroscience \& Biobehavioral Reviews, 35(5), 1219-1236.

doi:10.1016/j.neubiorev.2010.12.012

Loued-Khenissi, L., Pfeuffer, A., Einhäuser, W., \& Preuschoff, K. (2020). Anterior insula reflects surprise in value-based decision-making and perception. NeuroImage, 210, 116549. doi:10.1016/j.neuroimage.2020.116549

Lundstrom, B. N., Ingvar, M., \& Petersson, K. M. (2005). The role of precuneus and left inferior frontal cortex during source memory episodic retrieval. Neuroimage, 27, 824-834. doi:10.1016/j.neuroimage.2005.05.008

Makris, N., Goldstein, J. M., Kennedy, D., Hodge, S. M., Caviness, V. S., Faraone, S. V., Tsuang, M. T., Seidman, L. J. (2006). Decreased volume of left and total anterior insular lobule in schizophrenia. Schizophr. Res. 83(2-3), 155-171.

McCarthy, P. (2020). FSLeyes (Version 0.27.3). Zenodo. doi:10.5281/zenodo.1470761

McClure, S. M., Laibson, D. I., Loewenstein, G., \& Cohen, J. D. (2004). Separate neural systems value immediate and delayed monetary rewards. Science, 306, 503-507.

Menon, V. \& Uddin, L. Q. (2010). Saliency, switching, attention and control: A network model of insula function. Brain Structure \& Function, 214(5-6), 655-667. doi:10.1007/s00429010-0262-0 
VALUE NETWORK DURING ENCODING AND RETRIEVAL

Montague, P. R., King-Casas, B., \& Cohen, J. D. (2006). Imaging valuation models in human choice. Annu. Rev. Neurosci., 29, 417-448.

Morris, L. S., Kundu, P., Dowell, N., Mechelmans, D. J., Favre, P., Irvine, M. A., Robbins, T. W., Daw, N., Bullmore, E. T., Harrison, N. A., \& Voon, V. (2016). Fronto-striatal organization: Defining functional and microstructural substrates of behavioural flexibility. Cortex, 74, 118-133. doi:10.1016/j.cortex.2015.11.004

Murty, V. P., FeldmanHall, O., Hunter, L. E., Phelps, E. A., \& Davachi, L. (2016). Episodic memories predict adaptive value-based decision-making. Journal of Experimental Psychology, 145(5), 548-558.

Nagel, B. J., Herting, M. M., Maxwell, E. C., Bruno, R., \& Fair, D. (2013). Hemispheric lateralization of verbal and spatial working memory during adolescence. Brain and Cognition, 82(1), 58-68. doi:10.1016/j.bandc.2013.02.007

Nicolle, A., Klein-Flügge, M. C., Hunt, L. T., Vlaev, I., Dolan, R. J., \& Behrens, T. E. (2012). An agent independent axis for executed and modeled choice in medial prefrontal cortex. Neuron, 79(3), 607. doi:10.1016/j.neuron.2013.07.030

Nogueira, R., Abolafia, J. M., Drugowitsch, J., Balaguer-Ballester, E., Sanchez-Vives, M. V., \& Moreno-Bote, R. (2017). Lateral orbitofrontal cortex anticipates choices and integrates prior with current information. Nature Communications, 8, 14823. doi:10.1038/ncomms 14823

O’Doherty, J., Dayan, P., Schultz, J., Deichmann, R., Friston, K., \& Dolan, R. J. (2004). Dissociable roles of ventral and dorsal striatum in instrumental conditioning. Science, $304,452-454$. 
VALUE NETWORK DURING ENCODING AND RETRIEVAL

Padoa-Schioppa, C. \& Assad, J. A. (2006). Neurons in the orbitofrontal cortex encode economic value. Nature, 441(7090), 223-226. doi:10.1038/nature04676

Palminteri, S., Boraud, T., Lafargue, G., Dubois, B., \& Pessiglione, M. (2009). Brain hemispheres selectively track the expected value of contralateral options. Journal of Neuroscience, 29(43), 13465-13472.

Park, H. \& Kayser, C. (2019). Shared neural underpinnings of multisensory integration and trialby-trial perceptual recalibration in humans. eLife, 8, e47001. doi:10.7554/eLife.47001

Pauli, W. M., O’Reilly, R. C., Yarkoni, T., \& Wager, T. D. (2016). Regional specialization within the human striatum for diverse psychological functions. Proc. Natl. Acad. Sci. USA, 113(7), 1907-1912. doi:10.1073/pnas.1507610113

Peters, J. \& Büchel, C. (2009). Overlapping and distinct neural systems code for subjective value during intertemporal and risky decision making. Journal of Neuroscience, 29(50), 1572715734. doi:10.1523/jneurosci.3489-09.2009

Peters, J. \& Büchel, C. (2010). Neural representation of subjective reward value. Behav. Brain. Res., 213, 135-141.

Petrides, M. (2007). The orbitofrontal cortex: Novelty, deviation from expectation, and memory. Annals of the New York Academy of Sciences, 1121, 33-53.

Ploran, E. J., Nelson, S. M., Velanova, K., Donaldson, D. I., Petersen, S. E., \& Wheeler, M. E. (2007). Evidence accumulation and the moment of recognition: Dissociating perceptual recognition processes using fMRI. Journal of Neuroscience, 27(44), 11912-11924. doi:10.1523/jneurosci.3522-07.2007 
VALUE NETWORK DURING ENCODING AND RETRIEVAL

Poppenk, J., McIntosh, A. R., Craik, F. I., \& Moscovitch, M. (2010). Past experience modulates the neural mechanisms of episodic memory formation. The Journal of Neuroscience, 30(13), 4707-4716. doi:10.1523/JNEUROSCI.5466-09.2010

Ptak, R. (2011). The frontoparietal attention network of the human brain. The Neuroscientist, 18(5), 502-515. doi:10.1177/1073858411409051

Rangel, A., Camerer, C., \& Montague, P. R. (2008). A framework for studying the neurobiology of value-based decision making. Nature Reviews Neuroscience, 9(7), 545-556. doi:10.1038/nrn2357

Richter, F. R., Chanales, A. J., \& Kuhl, B. A. (2015). Predicting the integration of overlapping memories by decoding mnemonic processing states during learning. NeuroImage, 124, 323-335. doi:10.1016/j.neuroimage.2015.08.051

Ritchey, M., Wing, E. A., LaBar, K. S., \& Cabeza, R. (2013). Neural similarity between encoding and retrieval is related to memory via hippocampal interactions. Cerebral Cortex, 23(12), 2818-2828. doi:10.1093/cercor/bhs258

Rorden, C., Karnath, H., \& Bonilha, L. (2007). Improving lesion-symptom mapping. Journal of Cognitive Neuroscience, 19, 1081-1088. doi:10.1162/jocn.2007.19.7.1081

Roy, M., Shohamy, D., \& Wager, T. D. (2012). Ventromedial prefrontal-subcortical systems and the generation of affective meaning. Trends in Cognitive Sciences, 16(3), 147-156. doi:10.1016/j.tics.2012.01.005

Rushworth, M. F. S. \& Behrens, T. E. J. (2008). Choice, uncertainty and value in prefrontal and cingulate cortex. Nature Neuroscience, 11, 389-397. doi:10.1038/nn2066 
VALUE NETWORK DURING ENCODING AND RETRIEVAL

Rushworth, M., Noonan, M., Boorman, E., Walton, M., \& Behrens, T. (2011). Frontal cortex and reward-guided learning and decision-making. Neuron, 70(6), 1054-1069. doi:10.1016/j.neuron.2011.05.014

Sadaghiani, S. \& D’Esposito, M. (2015). Functional characterization of the cingulo-opercular network in the maintenance of tonic alertness. Cerebral Cortex, 25(9), 2763-2773. doi:10.1093/cercor/bhu072

Seeley, W. W., Menon, V., Schatzberg, A. F., Keller, J., Glover, G. H., Kenna, H., Reiss, A. L., \& Greicius, M. D. (2007). Dissociable intrinsic connectivity networks for salience processing and executive control. The Journal of Neuroscience, 27(9), 2349-2356. doi:10.1523/JNEUROSCI.5587-06.2007

Shadlen, M. N., \& Shohamy, D. (2016). Decision making and sequential sampling from memory. Neuron, 90(5), 927-939. doi:10.1016/j.neuron.2016.04.036

Shenhav, A., Barrett, L. F., \& Bar, M. (2013). Affective value and associative processing share a cortical substrate. Cognitive, Affective, \& Behavioral Neuroscience, 13(1), 46-59. doi:10.3758/s13415-012-0128-4

Shenhav, A., Rand, D. G., \& Greene, J. D. (2017). The relationship between intertemporal choice and following the path of least resistance across choices, preferences, and beliefs. Judgment and Decision Making, 12(1), 1-18.

Simmons, J., Nelson, L., \& Simonsohn, U. (2011). False-positive psychology: Undisclosed flexibility in data collection and analysis allows presenting anything as significant. Psychological Science, 22(11), 1359-1366. doi:10.1037/e519702015-014 
VALUE NETWORK DURING ENCODING AND RETRIEVAL

Smith, S. M., Jenkinson, M., Woolrich, M. W., . . \& Matthews, P. M. (2004). Advances in functional and structural MR image analysis and implementation as FSL. NeuroImage, 23. doi:10.1016/j.neuroimage.2004.07.051

Spalding, K. N., Schlichting, M. L., Zeithamova, D., Preston, A. R., Tranel, D., Duff, M. C., \& Warren, D. E. (2018). Ventromedial prefrontal cortex is necessary for normal associative inference and memory integration. The Journal of Neuroscience, 38(15), 3767-3775. doi:10.1523/JNEUROSCI.2501-17.2018

Stalnaker, T. A., Cooch, N. K., \& Schoenbaum, G. (2015). What the orbitofrontal cortex does not do. Nature Neuroscience, 18(5), 620-627. doi:10.1038/nn.3982

Stöttinger, E., Aichhorn, M., Anderson, B., \& Danckert, J. (2018). The neural systems for perceptual updating. Neuropsychologia, 112, 86-94.

doi:10.1016/j.neuropsychologia.2018.03.017

Strait, C. E., Sleezer, B. J., \& Hayden, B. Y. (2015). Signatures of value comparison in ventral striatum neurons. PLoS Biol., 13(6), e1002173. doi:10.1371/journal.pbio.1002173

Thirion, B., Pinel, P., Mériaux, S., Roche, A., Dehaene, S., \& Poline, J.-B. (2007). Analysis of a large fMRI cohort: Statistical and methodological issues for group analyses. NeuroImage, 35(1), 105-120. doi:10.1016/j.neuroimage.2006.11.054

Tom, S. M., Fox, C. R., Trepel, C., \& Poldrack, R. A. (2007). The neural basis of loss aversion in decision-making under risk. Science, 315(5811), 515-518. doi:10.1126/science.1134239

Tosoni, A., Corbetta, M., Calluso, C., Committeri, G., Pezzulo, G., Romani, G. L., \& Galati, G. (2014). Decision and action planning signals in human posterior parietal cortex during delayed perceptual choices. European Journal of Neuroscience, 39(8), 1370-1383. doi:10.1111/ejn.12511 
VALUE NETWORK DURING ENCODING AND RETRIEVAL

Tricomi, E., \& Lempert, K. M. (2015). Value and probability coding in a feedback-based learning task utilizing food rewards. Journal of Neurophysiology, 113(1), 4-13. doi:10.1152/jn.00086.2014

Uncapher, M. R. \& Wagner, A. D. (2009). Posterior parietal cortex and episodic encoding: Insights from fMRI subsequent memory effects and dual-attention theory. Neurobiology of Learning and Memory, 91(2), 139-154. doi:10.1016/j.nlm.2008.10.011

Valentin, V. V., Dickinson, A., \& O’Doherty, J. P. (2007). Determining the neural substrates of goal-directed learning in the human brain. Journal of Neuroscience, 27(15), 4019-4026. doi:10.1523/jneurosci.0564-07.2007

Wallis, J. D. (2007). Orbitofrontal cortex and its contribution to decision-making. Annual Review of Neuroscience, 30(1), 31-56. doi:10.1146/annurev.neuro.30.051606.094334

Wallis, G., Stokes, M., Cousijn, H., Woolrich, M., \& Nobre, A. C. (2015). Frontoparietal and cingulo-opercular networks play dissociable roles in control of working memory. Journal of Cognitive Neuroscience, 27(10), 2019-2034.doi:10.1162/jocn_a_00838

Walton, M. E., Chau, B. K., \& Kennerley, S. W. (2015). Prioritising the relevant information for learning and decision making within orbital and ventromedial prefrontal cortex. Current Opinion in Behavioral Sciences, 1, 78-85.

Watson, P., van Wingen, G., \& de Wit, S. (2018). Conflicted between goal-directed and habitual control, an fMRI investigation. eNeuro, 5(4), ENEURO.0240-18.2018. doi:10.1523/ENEURO.0240-18.2018

Wimmer, G. E., Braun, E. K., Daw, N. D., \& Shohamy, D. (2014). Episodic memory encoding interferes with reward learning and decreases striatal prediction errors. The Journal of Neuroscience, 34(45), 14901-12. 
VALUE NETWORK DURING ENCODING AND RETRIEVAL

Wimmer, G. E., Li, J. K., Gorgolewski, K. J., \& Poldrack, R. A. (2018). Reward learning over weeks versus minutes increases the neural representation of value in the human brain. The Journal of Neuroscience, 38(35), 7649-7666.

Wolosin, S. M., Zeithamova, D., \& Preston, A. R. (2013). Distributed hippocampal patterns that discriminate reward context are associated with enhanced associative binding. Journal of Experimental Psychology, 142(4), 1264-1276. doi:10.1037/a0033609

Woo, C.-W., Krishnan, A., \& Wager, T. D. (2014). Cluster-extent based thresholding in fMRI analyses: Pitfalls and recommendations. NeuroImage, 91, 412-419. doi:10.1016/j.neuroimage.2013.12.058

Worsley, K. J. (2001). Statistical analysis of activation images. In P. Jezzard, P. M. Matthews, S. M. Smith (Eds.), Functional MRI: An introduction to methods. Oxford University Press (pp. 251-270).

Wunderlich, K., Rangel, A., \& O’Doherty, J. P. (2009). Neural computations underlying actionbased decision making in the human brain. Proc. Natl Acad. Sci. USA, 106, 1719917204. doi:10.1073/pnas.0901077106

Yeo, B. T., Krienen, F. M., Sepulcre, J., Sabuncu, M. R., Lashkari, D., Hollinshead, M., . . \& Buckner, R. L. (2011). The organization of the human cerebral cortex estimated by intrinsic functional connectivity. Journal of Neurophysiology, 106(3), 1125-1165. doi:10.1152/jn.00338.2011

Zeithamova, D., Dominick, A. L., \& Preston, A. R. (2012). Hippocampal and ventral medial prefrontal activation during retrieval-mediated learning supports novel inference. Neuron, 75(1), 168-179. doi:10.1016/j.neuron.2012.05.010 
VALUE NETWORK DURING ENCODING AND RETRIEVAL

Zhang, S., \& Li, C. S. (2012). Functional connectivity mapping of the human precuneus by resting state fMRI. NeuroImage, 59(4), 3548-3562.

doi:10.1016/j.neuroimage.2011.11.023

Zhang, Y., Larcher, K. M., Misic, B., \& Dagher, A. (2017). Anatomical and functional organization of the human substantia nigra and its connections. eLife, 6, e26653. doi:10.7554/eLife.26653 